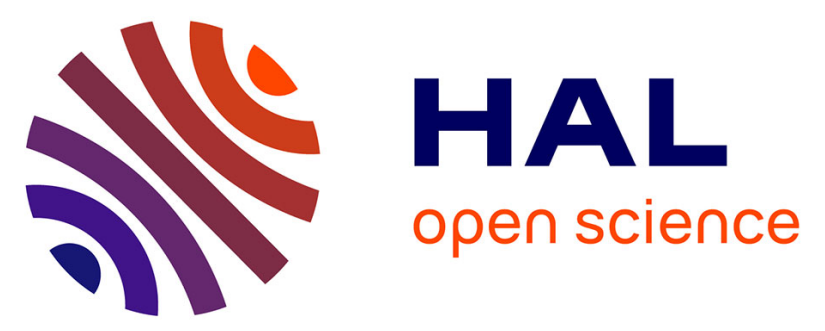

\title{
On the use of autocorrelation functions, permeability tensors, and computed tomography to characterise the anisotropy of diesel particulate filter materials
}

Dominique Bernard, Fabien Léonard, Erwan Plougonven, Giovanni Bruno

\section{- To cite this version:}

Dominique Bernard, Fabien Léonard, Erwan Plougonven, Giovanni Bruno. On the use of autocorrelation functions, permeability tensors, and computed tomography to characterise the anisotropy of diesel particulate filter materials. Philosophical Magazine, 2020, 100 (22), pp.2802-2835. 10.1080/14786435.2020.1798532 . hal-02947931

\section{HAL Id: hal-02947931 \\ https://hal.science/hal-02947931}

Submitted on 7 Oct 2020

HAL is a multi-disciplinary open access archive for the deposit and dissemination of scientific research documents, whether they are published or not. The documents may come from teaching and research institutions in France or abroad, or from public or private research centers.
L'archive ouverte pluridisciplinaire HAL, est destinée au dépôt et à la diffusion de documents scientifiques de niveau recherche, publiés ou non, émanant des établissements d'enseignement et de recherche français ou étrangers, des laboratoires publics ou privés. 
On the use of autocorrelation functions, permeability tensors, and computed tomography to characterise the anisotropy of diesel particulate filter materials

D. Bernard ${ }^{1}$, F. Léonard ${ }^{2}$, E. Plougonven ${ }^{3}$, G. Bruno ${ }^{2,4}$

1 - CNRS, Univ. Bordeaux, Bordeaux INP, ICMCB, UMR 5026, F-33600 Pessac, France

2 - BAM, Bundesanstalt für Materialforschung und -prüfung, 12205 Berlin, Germany

3 - University of Liege, Belgium

4 - University of Potsdam, Institute of Physics and Astronomy, 14476 Potsdam, Germany

Corresponding author: Dominique Bernard, ICMCB, 87 av. Schweitzer, F-33608 PESSAC CEDEX, FRANCE, Dominique.Bernard@icmcb.cnrs.fr 


\title{
On the use of autocorrelation functions, permeability tensors, and computed tomography to characterise the anisotropy of Diesel particulate filter materials
}

\begin{abstract}
We show how the combination of the spatial autocorrelation function and permeability calculations, applied to 3D X-ray computed tomography data, can yield quantitative information on the anisotropy of both meso-structure and fluid flow in Diesel Particulate Filter (DPF) materials, such as Cordierite and $\mathrm{SiC}$. It was found that both the degree of anisotropy, and the orientation of the permeability and meso-structure are similar, but not identical. We confirm that the morphological anisotropy of cordierite materials is weak, and clearly influenced by the extrusion process that determines the main direction of anisotropy.

Properties of the autocorrelation function are discussed and it is shown why estimating the characteristic lengths of real meso-structures (grain or "pore" size) is not possible. Finally, we show that the autocorrelation function applied on grey-level images can give a good estimate of the degree of anisotropy even with limited resolution.
\end{abstract}

Keywords: Anisotropy; Autocorrelation function; Computed tomography; Permeability tensor; Diesel particulates filter; Ceramics

\section{Introduction}

3D images such as the reconstruction of X-ray tomography data can provide a wealth of information on the internal structure of materials, but quantification of specific geometrical or topological characteristics linked to some bulk physical property is far from straightforward. It is well known that properties of the crystal lattice of such materials are anisotropic, but the degree of anisotropy of the macroscopic properties depends on the meso-structure (i.e. the arrangement of the material and the pore space). This study focuses on methods to quantify the differences in physical properties as a function of direction, i.e. their anisotropy, and how it can be linked to measures of anisotropy of the internal structure of the material. 
The methods to measure the anisotropy of meso-structures through image analysis have mostly originated from the study of 2D images to characterize deformed grains in the domains of geosciences [1] or metallurgy [2]. More recently, in the medical domain research aiming at characterizing the structure of trabecular bones has produced various secant-based methods to compute standardized histomorphometry parameters [3]. Such methods are based on shooting parallel lines at different angles in the object, and counting the number of traversals through the porous or solid phase versus the lengths of the segments. The most widely used is called Mean Intercept Length (MIL) [4], others include Intercept Segmentation Deviation (ISD) [5], or Line Fraction Deviation (LFD) [6]. There are also point-based methods, that perform local measurements (again by shooting lines) from different points in the image, such as Volume Orientation (VO) [7], Star Chord Length (SCL) [8], Star Volume Distribution (SVD) [9], or Star Length Distribution (SLD) [10]. The main drawback of these methods is that they require segmented images, i.e. images that have been processed to separate the background (the pore space) and the foreground (the bone in these works). The segmentation step can be a strong source of error for images with high level of noise or affected by acquisition artefacts. Methods, circumventing the need to segment and thus working directly on the grey-level images, have been investigated. Examples are the Fast Fourier Transform (FFT) for 2D radiographs [11], Gabor wavelets [12], or Tensor Scale Distribution (TSD) [13]. The main problem of these methods is their expensive computational time that makes their extension to 3D almost prohibitive. These practical limitations to 2D require a priori knowledge of a symmetry plane of the studied material to be unbiased. An attractive alternative, combining possibility to process grey-level images and computational efficiency, is the spatial Auto-Correlation Function $(\mathrm{ACF})[14,15]$. 
The ACF gives a similarity measure in the volume as a function of distance and direction. It consists in a cross-correlation of the image with itself. The ACF belongs to the family of the two-points correlation functions. These functions condense a lot of information about the morphology of the processed images, making then suitable for applications as different as advanced segmentation of 3D images of granular materials [16] or reconstruction of different realisations of similar heterogeneous materials [17]. In these examples, as in most of the applications, the studied materials are considered as isotropic, making the two-points correlation function depend on the distance only. Regarding the anisotropy estimate, autocorrelation has been compared with MIL by Wehrli et al. in $[18,19]$. For high resolution images of trabecular bones MIL is considered as the gold standard, but the authors favour autocorrelation because of its speed and lower sensitivity to noise. The ACF can be easily computed for 3D images after segmentation or not. As shown in this paper, when the full 3D ACF is analysed the different directions of anisotropy can be revealed without a priori. We focus on this method to quantify the anisotropy of Diesel Particulate Filter (DPF) materials from 3D reconstructions of X-ray tomography data and compare the results with the ones given by another approach based on their filtration properties.

Diesel Particulate Filter (DPF) materials are usually porous ceramics, often containing micro-cracks $[20,21]$. The reasons for this choice are manifold: i- they can be used at very high temperatures (typically around $800{ }^{\circ} \mathrm{C}$, but sometimes in excess of $\left.1000{ }^{\circ} \mathrm{C}\right)$; ii- they have very good thermal shock resistance in their temperature range of application (especially if micro-cracked); iii- they are inert to many gases and combustion products; iv- they can be manufactured with tailored porosity fraction.

They are typically produced by extrusion of a slurry into the desired filter shape, with successive sintering at high temperature $[22,23]$. This extrusion process causes 
anisotropy at both microscopic (grains, pores) and macroscopic (properties) levels. In other words, the crystallographic (grain orientation), meso-structural (pore space orientation), and material properties (mechanical, thermal, and filtration) acquire a preferential orientation along the extrusion axis. This orientation dependence extends at the component level, and is exacerbated by the cellular geometry of the filter.

Indeed, a dependence of material properties on direction has been reported at the component level (Young's modulus and thermal expansion, [24]) and at the material level (Coefficient of Thermal Expansion [25], Fracture toughness [26, 27], stress-strain curves [28]). Recently, an analysis of both X-ray refraction and computed tomography images [29-31] has shown that the orientation of the pore space is directly correlated with the anisotropy of the mechanical and thermal properties.

The pore space in these materials is totally interconnected and has a very complex geometry making its decomposition in representative pore elements difficult. Extracting relevant average properties allowing the use of models based on 'unit cells' containing voids of defined geometry $[32,33]$ is consequently questionable. Instead, we need to consider, as done in this work, approaches that tackle the problem of determining global properties of the materials using the complete 3D images.

Besides mechanical and thermal properties, certainly important for the structural stability of such DPFs, the functional properties (and especially filtration) are extremely relevant for their performance. Not much work is available in the open literature dealing with the effect of the meso-structure of such filters on the filtration properties. Relevantly for the present study, synthetic cordierite meso-structures have been investigated using X-ray tomography data in [34]; however, while global porosity fraction and permeability values have been obtained, no information about the permeability tensor orientation has been given. Otherwise, much work has been 
dedicated to numerical models of permeability and pressure drop, see e.g. [35], the latter being relevant for industrial applications.

While the meso-structure of these materials has been classically investigated by means of scanning electron microscopy (see e.g. $[27,36]$ ), since the resolution needed lies within the realm of SEM, a global view of the meso-structure is also needed, yet with the highest possible resolution. Optical microscopy $(\mathrm{OM})$ can yield relevant information, but is $2 \mathrm{D}$ and lacks resolution. On the contrary, X-ray computed tomography (CT) has the potential to disclose features in the bulk (3D analysis), with a resolution in between SEM and OM, and a large field of view, comparable to OM. As with other imaging techniques, if one is to go beyond qualitative assessment of the features of a structure, image processing algorithms are needed to extract quantitative information.

The scope of this work is to apply specific methods (Autocorrelation function; Permeability calculations) to 3D CT reconstructions in order to characterize the structural and filtration anisotropies. We consider samples extracted from filters of different materials (cordierites and silicon carbide) having similar porosity fraction but different arrangement of the pore space. We determine their permeability tensors as well as the autocorrelation function of the computed tomography reconstructions and use these results to quantify the anisotropy of the materials by two different methods. These global approaches have the advantage of being directly comparable with analytical and numerical global models, designed to extract or predict properties.

\section{Characterization methods}

\section{Computed Tomography}

X-ray computed tomography $(\mathrm{CT})$ is a non-destructive imaging technique based on 
differences in X-ray absorption from the multiple constituents of a specimen (different materials, densities, and/or features such as inclusions, pores, cracks, matrix). The specimen is placed between an X-ray source and a detector, then rotated so that a series of $2 \mathrm{D}$ radiographic images (usually thousands) can be acquired over a rotation of the specimen on $180^{\circ}$ or $360^{\circ}$. These $2 \mathrm{D}$ radiographic images are then processed so that a $3 \mathrm{D}$ digital reconstruction of the specimen can be obtained. The reconstructed volume is a 3D image composed of 3D pixels with a grey value representing the $\mathrm{X}$-ray attenuation in the corresponding volume in the specimen. Therefore, to be able to resolve a certain feature, two conditions are required: first, the feature must be significantly larger than the pixel size of the reconstructed volume, and second, there should be enough contrast in the grey values corresponding to the different features of interest.

\section{Laboratory X-ray computed tomography}

Laboratory XCT experiments were performed on a GE v|tome|x L 300/180 equipped with a $180 \mathrm{kV}$ source, a tungsten transmission target (actual focal spot size below $2 \mu \mathrm{m}$ as determined with JIMA test pattern RTC02), and a GE $2000 \times 2000$ pixel DXR-250 detector. The source was operated at a voltage of $60 \mathrm{kV}$ and a current of $170 \mu \mathrm{A}$. The sample projections were taken at 1500 angular positions per $360^{\circ}$ (increments of $0.24^{\circ}$ ), and an exposure time of 3 seconds. The samples were placed $8 \mathrm{~mm}$ downstream from the source, with a source-detector distance of $800 \mathrm{~mm}$, and a binning of $2 \times 2$ pixels, so that the effective magnification obtained was 50. The resulting pixel size was $4.0 \mu \mathrm{m}$ and the scan time $1 \mathrm{~h} 15 \mathrm{~min}$. In order to ensure the projection of the entire sample, pieces of $2 \times 2$ cells were cut out of the honeycomb filter.

\section{Synchrotron radiation X-ray computed tomography}

Synchrotron-CT measurements were performed at the beamline BAMline [37, 38] at the 
synchrotron source BESSY II of the Helmholtz-Zentrum Berlin für Materialien und Energie (HZB), Berlin, Germany. The beam energy was set to $15 \mathrm{keV}$, in order to achieve optimal contrast for the investigated porous filter structures. A PCO4000 CCD camera (4008 $\times 2672$ pixels) was used in combination with an Optique Peter microscope equipped with a $\mathrm{CdWO}_{4}$ scintillator and a 10 -fold magnifying objective, resulting in a pixel size of $0.434 \mu \mathrm{m}$. A series of 3200 projections were acquired per $180^{\circ}$ rotation, with a $4 \mathrm{~s}$ exposure. A small sample-detector distance of $15 \mathrm{~mm}$ was set to ensure a limited scattering range of refractive edge artefacts and the entrance slits were narrowed to the field of view $\left(\mathrm{FoV}=1.7 \times 1.1 \mathrm{~mm}^{2}\right)$ in order to avoid detector backlighting $[39,40]$. To avoid the sample size exceeding the FoV, small splinters of less than $1.5 \mathrm{~mm}$ size were prepared from each sample, containing just one crossing region and one protruding wall of the filter.

\section{Autocorrelation Function}

The autocorrelation function of a real-valued image I is defined as:

$$
\mathrm{ACF}_{\mathrm{I}}(\mathbf{v})=\langle\mathrm{I}(\mathbf{u}) \cdot \mathrm{I}(\mathbf{u}+\mathbf{v})\rangle
$$

where $\mathrm{I}(\mathbf{u})$ is the value of the image at the position specified by the vector $\mathbf{u}, \mathrm{I}(\mathbf{u}+\mathbf{v})$ the value of the image at the position $\mathbf{u}$ plus the lag vector $\mathbf{v}$, and $<>$ is the averaging operator over the support of the image. With this expression $\operatorname{ACF}_{I}(\mathbf{v})$ is the expectation of the product of the image I with a copy of I that is offset by $\mathbf{v}$.

Most of the time what is called autocorrelation function does not correspond to expression (1), but to the NACFC, the normalised autocorrelation function of the centred image $(\mathrm{I}(\mathbf{u})-\langle\mathrm{I}\rangle)[41]$, noted $\mathrm{C}_{\mathrm{I}}(\mathbf{v})$ :

$$
\mathrm{C}_{\mathrm{I}}(\mathbf{v})=\frac{\operatorname{ACF}_{\mathrm{I}-\langle\mathrm{I}\rangle}(\mathbf{v})}{\operatorname{Var}(\mathrm{I})}=\frac{\langle[\mathrm{I}(\mathbf{u})-\langle\mathrm{I}\rangle] \cdot[\mathrm{I}(\mathbf{u}+\mathbf{v})-\langle\mathrm{I}\rangle]\rangle}{\left\langle[\mathrm{I}(\mathbf{u})-\langle\mathrm{I}\rangle]^{2}\right\rangle}
$$


where $\operatorname{Var}(\mathrm{I})$ is the variance of the image I.

Both expressions ( 1 and 2) quantify, as a function of $\mathbf{v}$, the correspondence of I with its copy offset by $\mathbf{v}$. A maximum always occurs for $\mathbf{v}=\mathbf{0}$ and, in case of a periodic image, for any $\mathbf{v}=\mathbf{l}_{\mathbf{p}}, \mathbf{l}_{\mathbf{p}}$ being the lattice vectors defining the periodic cell. For all other values of $\mathbf{v}, \mathrm{ACF}_{\mathrm{I}}$ and $\mathrm{C}_{\mathrm{I}}$ contain information about the effect of distance $(\|\mathbf{v}\|)$ and orientation $(\mathbf{v} /\|\mathbf{v}\|)$ on the correlation of the image I with itself. This encouraged some authors to attempt extracting dimensional information (grain size for instance) from $\mathrm{ACF}_{\mathrm{I}}$ or $\mathrm{C}_{\mathrm{I}}$, but with a limited success for real or realistic 3D images of materials (see [41] for a review on usage in geosciences, and Appendix A for a note concerning the use of $\mathrm{C}_{\mathrm{I}}$ for this purpose). On the contrary, anisotropy estimates seem to be more achievable even if some aspects remain unclear [42, 43].

To illustrate the possibilities offered by the use of the autocorrelation function, we consider the example of the dense packing of ellipsoidal particles represented by Fig. 1.

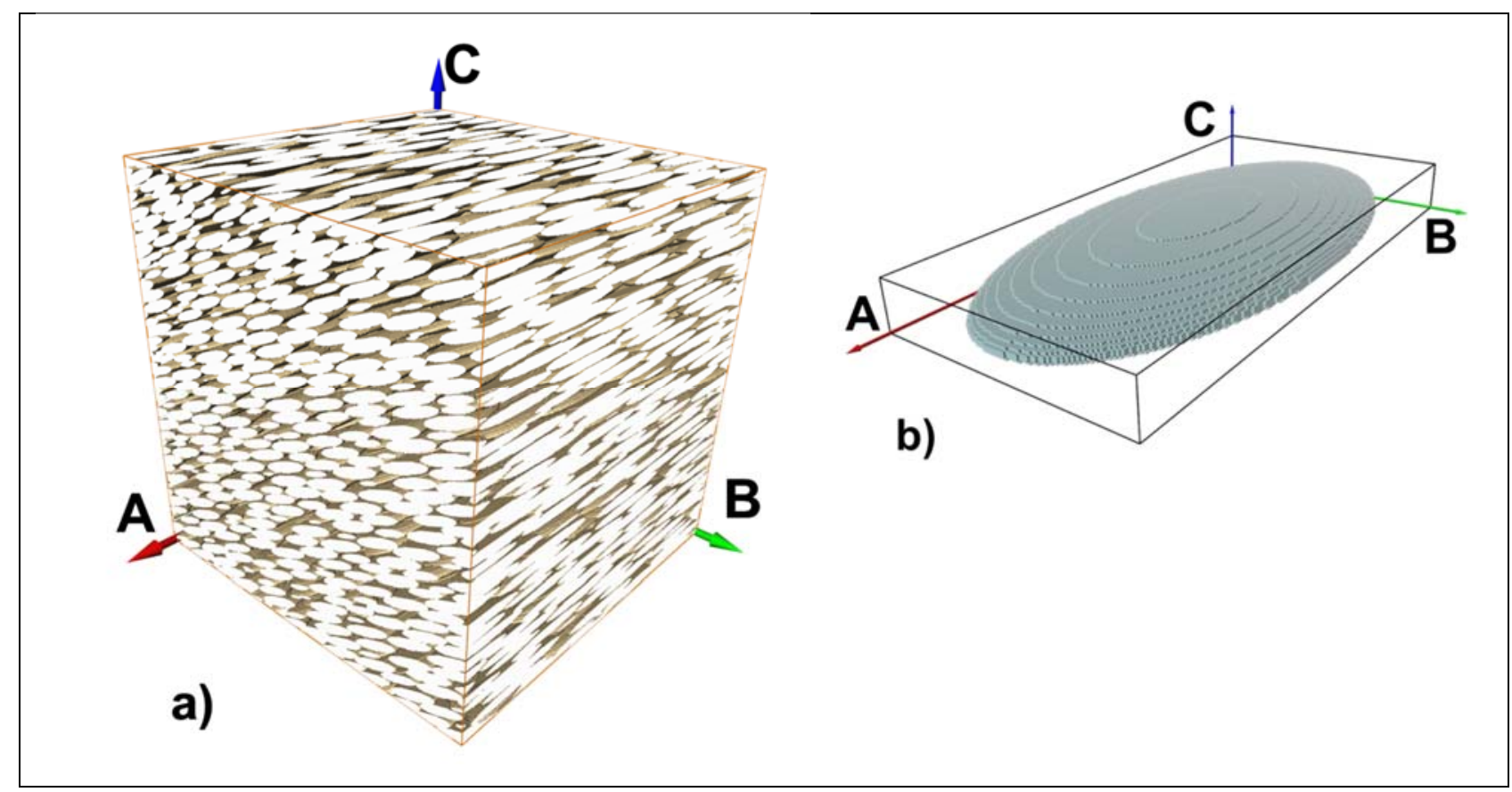

Figure 1: About 1500 of the ellipsoids represented in b) were randomly distributed in a cube to obtain the close packing shown in a). 
The starting point was a random close packing of $10^{5}$ non-overlapping spheres produced using a generator based on [44] (see Appendix A for more details). All the spheres, having the same density (set equal to 1) and the same diameter (set equal to $2.299510^{-2}$ in relative units to the cube edge), were placed within a unit cube (edges equal to one). Periodic boundary conditions were applied and the resulting packing had a theoretical porosity fraction equal to 0.3634 . The positions of the sphere centres were modified by dividing the $\mathrm{B}$ coordinate (green axis Fig. 1) by 2, and the $\mathrm{C}$ coordinate (blue axis) by 10. An ellipsoidal particle (Fig. 1.b) was then placed at each modified centre position, and a rotation of $23^{\circ}$ around the A axis (red axis) applied. Finally, the discrete binary image $\mathrm{I}_{\mathrm{El}}\left(1000^{3}\right.$ pixels $)$ presented in Fig. 1.a was generated from the central region of this ellipsoid packing.

The resulting image is simpler than 3D images of real materials because:

- all particles are identical (geometry, density, and orientation),

- there is no overlap between particles

- all distances between near neighbours are different (because of randomness), but similar (because of compactness).

On the other hand, this image is much more relevant than examples regularly used in the literature (single 2D/3D objects or loose aggregates $[41,43,45]$ ) as it represents a very dense random packing (porosity fraction of the final discretised packing $=0.3279)$ with very anisotropic particles.

The corresponding NACFC, $\mathrm{C}_{\mathrm{IE}}(\mathbf{v})$, was computed in about 3 minutes on a middle-range PC using a classic Fast Fourier Transform algorithm [46]. $\mathrm{CIEI}_{\mathrm{IE}}(\mathbf{v})$ is a 3D real-valued function with complicated visual representation. For isotropic images, it is 
sufficient to represent $\mathrm{C}_{\mathrm{IEI}}(\mathrm{r} . \mathbf{w})$ as a function of $\mathrm{r}$ for any direction $\mathbf{w}$ (see examples in Appendix A).

For anisotropic images, 1D or 2D representations can be misleading if the anisotropy principal directions are not known [42]. This is the reason why approaches to the determination of the pore space anisotropy such as the one presented in [30] do not fully describe the orientation of the meso-structure. In Fig. 2.a, the shape of $\mathrm{C}_{\mathrm{IEI}}(\mathbf{v})$ is deformed because the axis $\mathrm{C}$, perpendicular to the section, is not a principal direction of anisotropy. In Fig. 2.b, the axis perpendicular to the section is A, a principal direction of anisotropy, and the 2D image is not deformed.

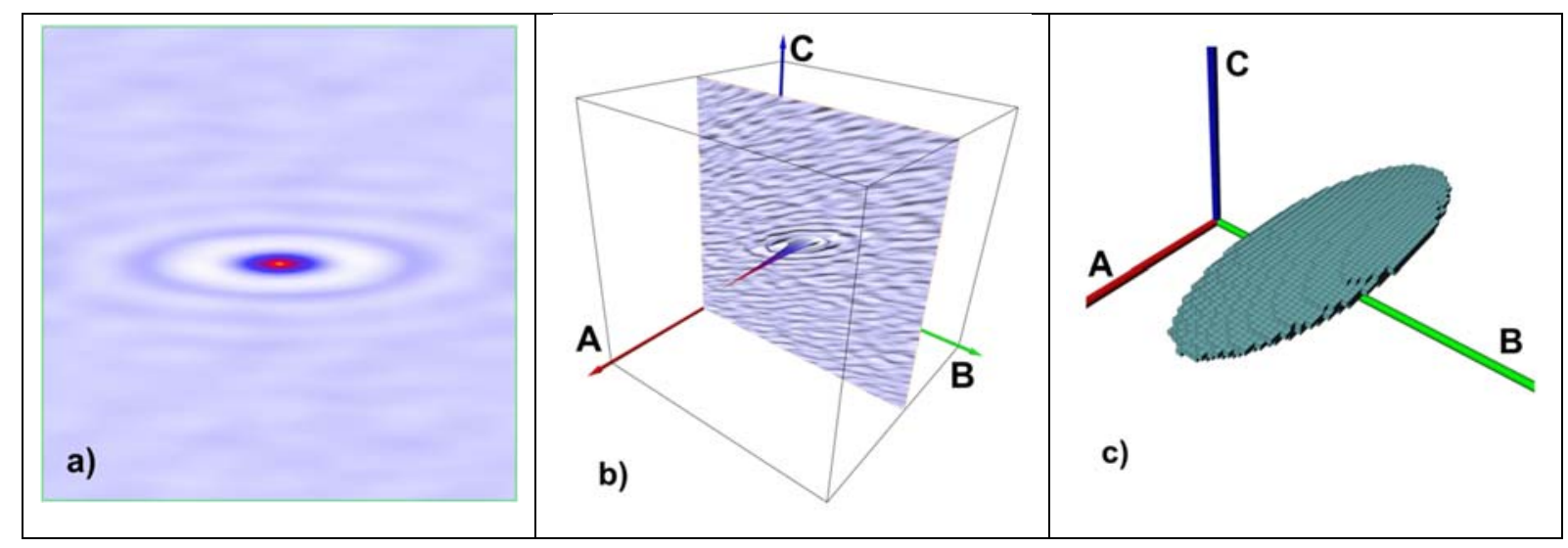

Figure 2: Three different representations giving a partial view of $C_{I E I}(v)$ : a) Section in the plane $A B$; b) Section in the plane BC as a colour-height map; c) Volume $V_{I E I}(0.5)$ composed by all the pixels having a correlation value $c \geq 0.5$.

Consequently, determining the principal directions of anisotropy appears mandatory before any further analysis. For that purpose, we developed the following procedure: first, $\mathrm{V}_{\mathrm{IEI}}(\mathrm{c})$, the volume made by the pixels with a correlation function (or its normalised version) value larger than a given threshold $\mathrm{c}(\mathrm{c}=0.5$ for the example given in Fig. 2.c), is built. Second, the tensor of inertia of this volume is computed. Third, $v_{1}$, $v_{2}, v_{3}$, the principal directions of this tensor are computed, giving the three principal directions of anisotropy. 
For each principal direction of anisotropy, it is then easy to represent the evolution of NACFC as a function of the lag along this direction. The resulting curves are presented Fig. 3 for the image IEl.
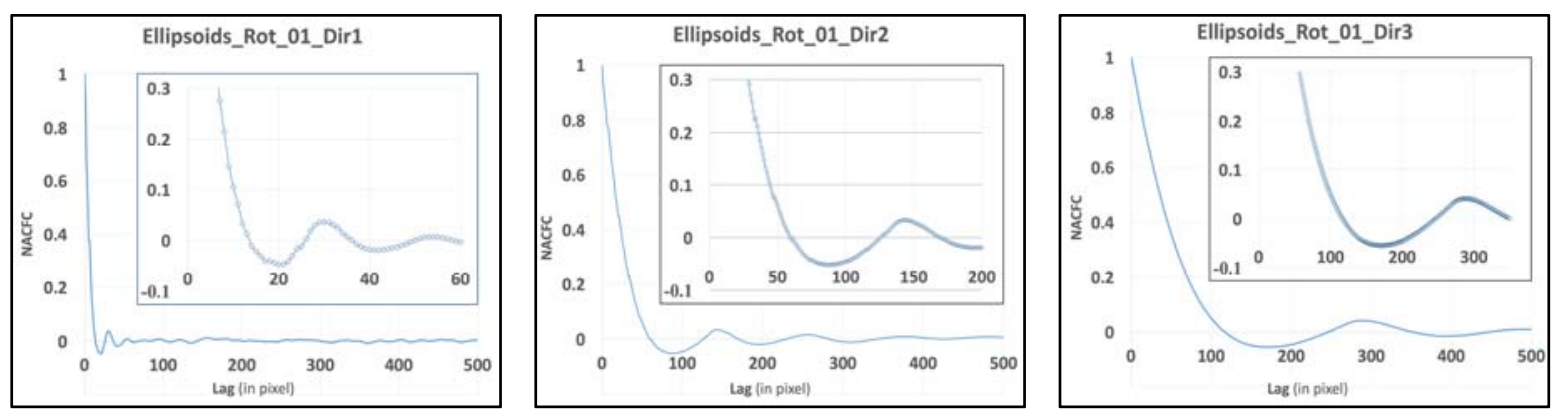

Figure 3: Evolutions of NACFCIEl along the three directions of anisotropy (from left to right: $\left.v_{1}, v_{2}, v_{3}\right)$.

Well defined minima and maxima are visible for the three directions. The lags corresponding to the minima (La) indicate the offset for which the level of anticorrelation is maximum, and the lags corresponding to the maxima $(\mathrm{Lc})$ indicate the offset for which the level of correlation is maximum. La and Lc are clearly different for the three directions of anisotropy. Nonetheless, they cannot be interpreted in terms of characteristic dimensions of the image components (grains size or grains distances, see Appendix A), and we must refrain from using their values for anisotropy characterisation. Furthermore, in most real cases La and Lc are not even detectable, as exemplified by the materials studied in this work.

Instead, the use of the properties of $\mathrm{V}_{\mathrm{IEI}}(\mathrm{c})$ should be favoured. The principal directions of anisotropy are the principal directions of its tensor of inertia, and we propose to estimate the degree of anisotropy DA as the ratio of the larger to the smaller moment of inertia with respect to the directions of anisotropy (DA_Inertia), or the ratio of the major to the minor length axis of the ellipsoid (DA_Ellips) having the same tensor of inertia as $\mathrm{V}_{\mathrm{IEI}}(\mathrm{c})$ (see Appendix B). 
Applied to the volume $\mathrm{V}_{\text {IEI }}(0.5)$ visualised Fig. 2.c, this leads to the two following values:

- DA_Inertia $=4.62$

- DA_Ellips $=8.66$

Interestingly, the fact that DA_Ellips > DA_Inertia implies that the shape of the ellipsoid $\mathrm{V}_{\mathrm{IEI}}(\mathrm{c})$ is closer to a disc than to a needle (see Appendix B).

A priori, both quantities are relevant, and it is only in the frame of a comparison between different methods for estimating the degree of anisotropy that a choice between them can be done. In this work, comparison is done with the values extracted from the permeability tensor computed from binary 3D images.

\section{Computation of permeability tensor}

In his seminal experimental work, Darcy [47] showed that when water is flowing through sand filters there is a linear relation between the flux and the pressure drop divided by the thickness of the filter. The coefficient of proportionality is the hydraulic conductivity. It comprises two terms: the first, viscosity, depends on the fluid, and the second, permeability, depends on the porous medium. More recently, several theoretical derivations of Darcy's law have been proposed leading to the vector form that is now generally used:

$$
\mathbf{V}=-\frac{\mathbf{K}}{\mu} \cdot \boldsymbol{\nabla p}
$$

where $\mathbf{V}$ is Darcy's velocity $\left(\mathrm{m} \mathrm{s}^{-1}\right), \mathbf{K}$ the permeability tensor $\left(\mathrm{m}^{2}\right), \mu$ the fluid dynamic viscosity $\left(\mathrm{kg} \mathrm{m}^{-1} \mathrm{~s}^{-1}\right), \nabla$ the gradient operator, and $\mathrm{p}$ the fluid pressure $\left(\mathrm{kg} \mathrm{m}^{-1} \mathrm{~s}^{-2}\right)$.

If, at the pore scale, the fluid flow is driven by Stokes' equations, Eq. 3 can be derived 
by change of scale using volume averaging [48]. With this method we not only derive Eq. 3, but also establish conditions for Darcy's equation to be valid. In so doing, we introduce the concept of REV (Representative Elementary Volume, i.e. the smallest volume over which the computed permeability tensor will be representative of the porous material), and construct a partial differential problem (the closure problem). The solution of this problem can be used to compute the permeability tensor directly from the local geometry [49]. The closure problem can be written as:

$$
-\nabla \mathbf{d}+\nabla^{2} \mathbf{D}+\mathbf{I}=0 ; \boldsymbol{\nabla} \cdot \mathbf{D}=0
$$

where $\mathbf{I}$ is the identity tensor, $\mathbf{d}$ an unknown vector and $\mathbf{D}$ an unknown tensor. This set of equations, very similar to Stokes equations, is completed by the following boundary conditions:

$$
\mathbf{D}=0 \text { at } \mathrm{A}_{\mathrm{sl}} ; \mathbf{d}\left(\mathrm{r}+\mathrm{l}_{\mathrm{i}}\right)=\mathbf{d}(\mathrm{r}) ; \mathbf{D}\left(\mathrm{r}+\mathrm{l}_{\mathrm{i}}\right)=\mathbf{D}(\mathrm{r}), \mathrm{i}=1,2,3
$$

where $A_{s l}$ is the solid-liquid interface. The porous medium is supposed to be periodic, and $\left(l_{i}, i=1,2,3\right)$ are the components of the lattice vector defining the periodic cell.

From D, the permeability tensor can be expressed as:

$$
\mathbf{K}=\varepsilon\langle\mathbf{D}\rangle^{l}
$$

where $\varepsilon$ is the global porosity fraction, and $\langle-\rangle^{1}$ is the notation for the averaging operator applied to the pore space only (see [48] for details).

This change of scale is possible when the sample is large enough to be representative of the porous medium. The $3 \mathrm{D}$ image is then an $\mathrm{REV}$, and periodic boundary conditions (Eq. 5) can be used without noticeable perturbations of the closure problem solution [50]. Consequently, when the conditions for the change of scale are 
verified, it is possible to compute the permeability tensor of a porous sample directly from a segmented 3D image by solving the closure problem (Eq. 4 to 6 ).

From Eq. 4 to 6 it is obvious that, through the boundary condition at the solidliquid interface $\mathrm{Asl}_{\mathrm{sl}}$, the permeability tensor is an implicit function of the geometry at the meso-scale only. Simplifying this implicit function, for instance to a reduced set of measureable characteristic parameters, is the object of a longstanding and continuous effort of research with rather limited advances [51, 52].

For many materials, direct measurement of permeability in a single direction is arduous, and estimating the full permeability tensor is generally impossible. Combining modern imaging techniques to acquire high quality 3D images describing the local meso-structure and efficient numerical code to solve the closure problem, a good approximation of the complete permeability tensor can be obtained. Although in practical applications solving the closure problem is always computationally demanding, this approach produces a relevant characteristic for anisotropic media that is experimentally unreachable.

In this work the closure problem is solved using an enhanced version of permea3D, a code developed at ICMCB (Bordeaux, France) and presented in [49]. In the frame of this work, the parallelization for very large 3D images was implemented.

\section{Diesel particulate filter materials and their meso-structure}

A total of five materials were investigated, four are cordierites (named Cord1 to Cord4) and one is $\mathrm{SiC}$. Cordierite materials were provided by Corning Incorporated (Corning, NY, USA) and Oak Ridge National Laboratory (Oak Ridge, TN, USA). All of them are extracted from larger honeycomb filters, all produced by the extrusion process mentioned above. In the case of cordierite, raw materials consisted of $\mathrm{SiO}_{2}, \mathrm{MgO}$, hydrated $\mathrm{Al}_{2} \mathrm{O}_{3}$, and $\mathrm{Al}_{2} \mathrm{Si}_{2} \mathrm{O}_{5}(\mathrm{OH})_{4}($ Caolin). Different porosity fractions were 
achieved by adding different amounts of pore formers. Sintering was made at temperatures in excess of $1300{ }^{\circ} \mathrm{C}$, with successive cooling at rates below $1{ }^{\circ} \mathrm{C} / \mathrm{min}$ in $\mathrm{N}_{2}$-enriched air. SiC filter materials were commercially available. Porosity fraction was determined by mercury intrusion with an Autopore 6520 Micromeritics, Norcross, USA. Porosity fraction values are reported in Table 1, together with the results of image analysis of computed tomography data (see below).

Table 1: Characteristics of the 3D binary sub-volumes. Pixel size (PS, in $\mu \mathrm{m})$, dimensions along the $A, B$ and $C$ axes (NA, NB, NC, in pixels), porosity measured from the CT images ( $\left.\phi_{C T}\right)$ of the sub-volumes, and porosity obtained by mercury porosimetry $\left(\phi_{\mathrm{Hg}}\right)$ on samples of the same materials.

\begin{tabular}{|c||c|c|c|c|c|c|c|c|}
\hline \hline Sample & IEI $_{\text {El }}$ & Cord1 & Cord2 & Cord3.1 & Cord3.2 & Cord4.1 & Cord4.2 & SiC \\
\hline \hline PS $(\boldsymbol{\mu m})$ & 1 & 4.00 & 4.00 & 0.868 & 0.868 & 0.868 & 0.868 & 0.868 \\
\hline NA & 1000 & 565 & 750 & 930 & 720 & 537 & 950 & 838 \\
\hline NB & 1000 & 755 & 650 & 1090 & 1140 & 1047 & 1110 & 1010 \\
\hline NC & 1000 & 58 & 99 & 275 & 240 & 260 & 250 & 390 \\
\hline$\phi$ Ст & 0.33 & 0.53 & 0.47 & 0.49 & 0.51 & 0.68 & 0.68 & 0.41 \\
\hline$\phi_{\mathrm{Hg}}$ & - & 0.52 & 0.48 & 0.50 & 0.50 & 0.65 & 0.65 & 0.38 \\
\hline
\end{tabular}

Samples Cord3, Cord4, and $\mathrm{SiC}$ were scanned on the synchrotron beamline BAMline, BESSY II, HZB Berlin, Germany; 2D slices of their corresponding reconstructions are shown in Fig. 4. Cordierite samples Cord1 and Cord2 were scanned in a laboratory CT system at BAM (see section on computed tomography), and their image size (as well as the field-of view) is much larger (see caption of Figure 4). For comparison purposes, slices of Cord1 and Cord 2 carry an inset with the same field of view as slices of Cord3, Cord4, and $\mathrm{SiC}$.

Qualitatively, Cord1 to Cord3 are similar materials, as they have similar chemical composition, pore former content and sintering schedule. In fact, they show similar pore space features: "pore elements" (intended as the dark objects visible in the CT slices) are sharp-edged and have a wide size distribution (accepting the legacy of the 
classic approach of measuring the pore size distribution by means of mercury porosity).

On the contrary Cord4 possesses much smoother pore/material interfaces and a larger porosity fraction (though finer pore space features). For SiC, traces of the powder particles composing the final material are clearly visible (analogous to IE1); correspondingly, the "pore elements" have a rounded shape. The total porosity fraction is lower than for the cordierite samples.
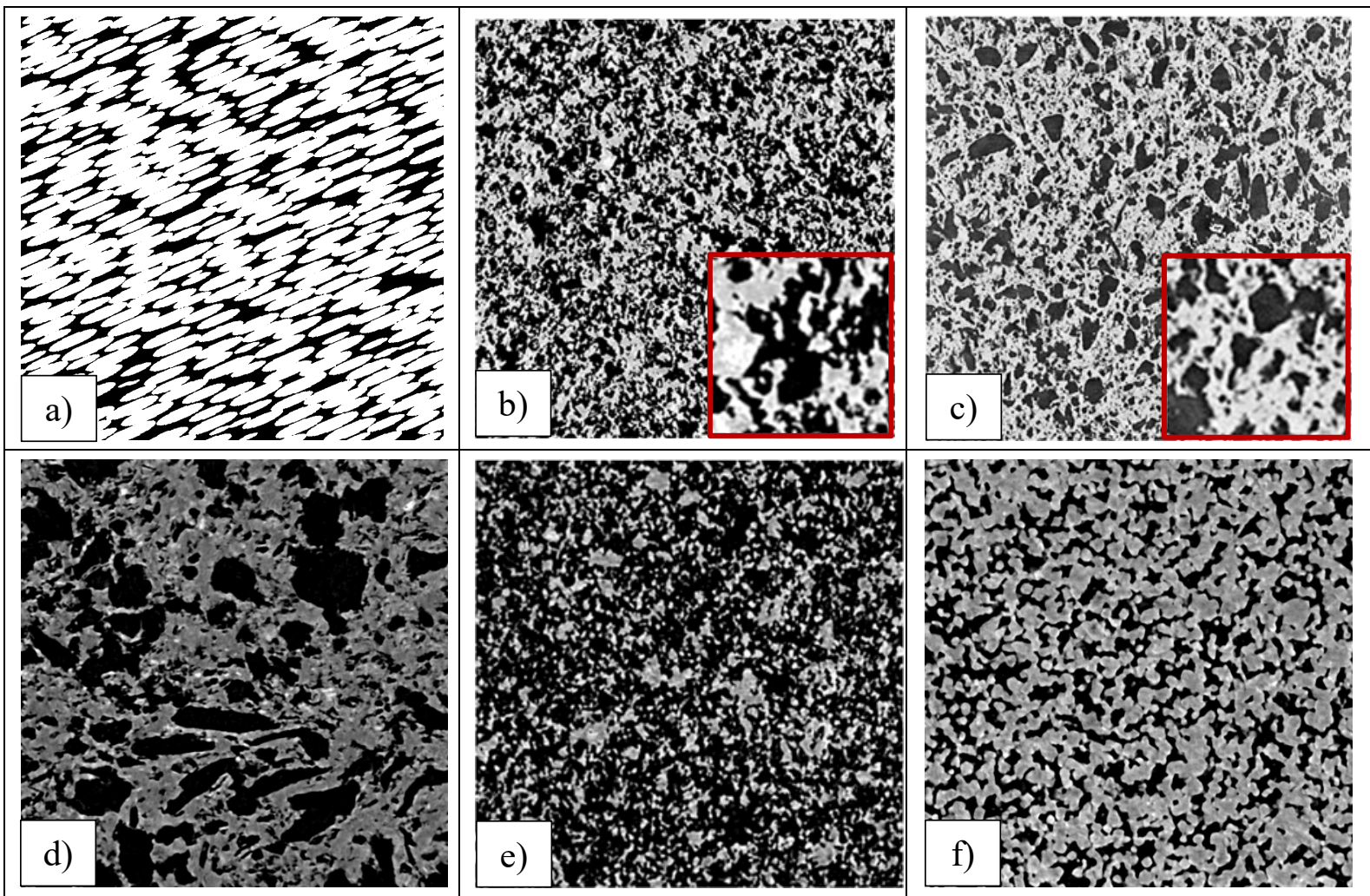

Figure 4: Overview of DPF samples under investigation. (a) IEl: Image size 1000 x 1000 pixels; (b) Cord1: Image size $2.18 \times 2.18 \mathrm{~mm}^{2}$, inset size $0.31 \times 0.31 \mathrm{~mm}^{2}$; (c) Cord2: Image size $2.18 \times 2.18 \mathrm{~mm}^{2}$, inset size $0.31 \times 0.31 \mathrm{~mm}^{2}$; (d) Cord3: Image size $0.31 \times 0.31$ $\mathrm{mm}^{2}$; (e) Cord4: Image size $0.31 \times 0.31 \mathrm{~mm}^{2}$; (f) SiC: Image size $0.31 \times 0.31 \mathrm{~mm}^{2}$. Interestingly, the homogeneity of the "pore elements" visible in the CT slices is higher for SiC and Cord4 than for Cord1 to Cord3. This is due to the fact that the $\mathrm{SiC}$ sample was probably manufactured using a sieved $\mathrm{SiC}$ powder (i.e., with narrow 
particle size distribution), and Cord4 was manufactured with smaller pore former particles than in the case of Cord1 to Cord3.

No clear global orientation of the pore space is visible from the CT slices.

\section{Results}

\section{Analysis of Computed Tomography Data}

The slices presented in Fig. 4 are extracted from the five 3D grey-level volumes used in this work. Their characteristics are given in Table 2; PS is the pixel size, in $\mu \mathrm{m}$, and $\mathrm{NA}, \mathrm{NB}, \mathrm{NC}$ the dimensions of the reconstructed volumes in pixels. In the first column of Fig. 5, 3D renderings of two of these volumes are presented.

Table 2: Characteristics of the 3D reconstructed volumes. PS is the pixel size, and NA, $N B, N C$ are the dimensions (in pixel) of the volumes along the three directions.

\begin{tabular}{||c|c|c|c|c|c||}
\hline Sample & Cord1 & Cord2 & Cord3 & Cord4 & SiC \\
\hline \hline PS $(\boldsymbol{\mu} \boldsymbol{m})$ & 4.00 & 4.00 & 0.434 & 0.434 & 0.434 \\
\hline NA & 1000 & 860 & 3201 & 3001 & 2501 \\
\hline NB & 800 & 820 & 2701 & 2101 & 2701 \\
\hline NC & 200 & 240 & 2672 & 2672 & 2672 \\
\hline
\end{tabular}

The study of the anisotropy requires a unique definition of the sample orientation. In this work we used reference axes related to the materials processing: A is along the wall length (red), $\mathrm{B}$ is in the extrusion direction (green), and $\mathrm{C}$ is normal to the wall (blue). Since sample positioning for the tomographic acquisitions was not precise enough, the volumes have been numerically realigned with the A, B, C axes.

For permeability and NACFC calculations domains fully immersed in the porous sample are necessary. They were obtained by extracting cuboid sub-volumes with boundaries near the interfaces between the porous material and the free fluid zones. In the middle column of Fig. 5 the boundaries of the sub-volumes (in black) are visible through the pore space of Cord2 (top) and Cord3 (bottom). Due to the peculiar 
shape of samples Cord3 (Fig. 5) and Cord4, it was possible to extract two overlapping sub-volumes with the zone corresponding to the intersection of two walls in common. These sub-volumes are labelled Cord3.1, Cord3.2, Cord4.1, and Cord4.2 hereafter.

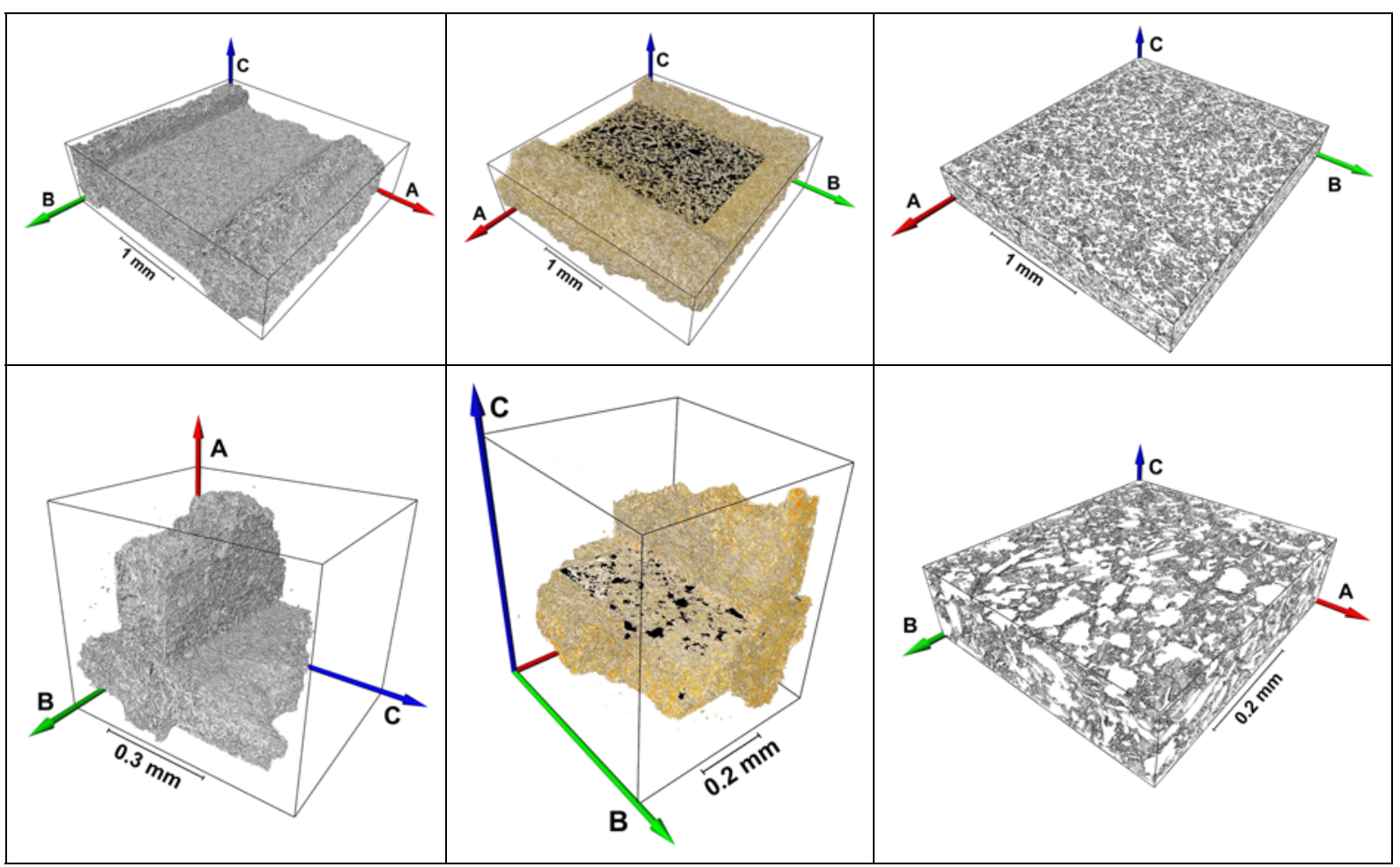

Figure 5: Visualization of process going from the initial reconstructed CT data to the binary sub-volumes used for computations. Top: sample Cord2, bottom: sample Cord3. First column: $3 D$ rendering of the initial volumes. Second column: $3 D$ rendering of the complete volumes after alignment with the processing axes (in yellow). The selected sub-volumes that were used for the calculations are visible in black. Third column: $3 D$ rendering of sub-volumes fully contained within the porous material.

Since permeability computations require binary images, the sub-volumes were segmented following the procedure exposed by Bernard et al. [42]: first, we filtered the grey-level images using an in-house developed anisotropic diffusion filter. The histograms of the filtered images presented two well identified peaks separated by a plateau. The grey-level situated in the middle of the two peaks was used as threshold 
value for binarization. Finally, considering the very large sizes (in terms of number of pixels) of the sub-volumes from the synchrotron acquisitions, they were sub-sampled by a factor of two.

Table 1 summarises the characteristics of the seven binary sub-volumes (plus the synthetic image presented Fig. 1) that are used for computations: pixel size (PS, in $\mu \mathrm{m})$, dimensions along the $\mathrm{A}, \mathrm{B}$ and $\mathrm{C}$ axes (NA, NB, NC, in pixels), and porosity fraction $(\phi \mathrm{CT})$. For comparisons, the porosity fraction values obtained by mercury porosimetry $(\phi \mathrm{Hg})$ are also given. Values obtained through the two methods are in good agreement.

\section{Autocorrelation functions}

The results detailed in this section were obtained for the binary images (solid/pore space) used for permeability computations. This ensures full and consistent comparison between the methods. It is of importance to notice that the NACFC can be computed from grey-level images as easily as from binary images. The differences between the two approaches are discussed in the last section of this paper.

For each of the eight binary sub-volumes (Table 1) the NACFC was computed and the volumes $V_{I}(c)$ were built. The latter contain all the pixels for which the NACFC is larger than the threshold $\mathrm{c}$. The threshold $\mathrm{c}$ needs to be large enough to avoid $\mathrm{V}_{\mathrm{I}}(\mathrm{c})$ having an irregular shape [42], but small enough to have a sufficient number of pixels within $\mathrm{VI}(\mathrm{c})$. The NACFC vary rapidly for small lags (Fig. 6) and, when pixels are large, the volumes $\mathrm{V}_{\mathrm{I}}(\mathrm{c})$ might be poorly discretized (composed of a small number of pixels) and subjected to important changes of shape for relatively small variations of $\mathrm{c}$. Consequences of this discretization effect on the degree of anisotropy are analysed in the corresponding section. To minimize both problems, $\mathrm{V}_{\mathrm{I}}(\mathrm{c})$ for $\mathrm{c}$ between 0.05 and 0.5 were considered. 
In Fig. 6 the values of the NACFC are plotted along the principal directions of anisotropy (major axis of the equivalent ellipsoid) of the volumes $\mathrm{V}_{\mathrm{I}}(0.2)$ presented in the insets. As indicated when presenting the autocorrelation function, the parameters La and $\mathrm{Lc}$ are not detectable, except for $\mathrm{SiC}$, a material having a meso-structure similar to a sphere-packing with large overlaps (see Appendix A).

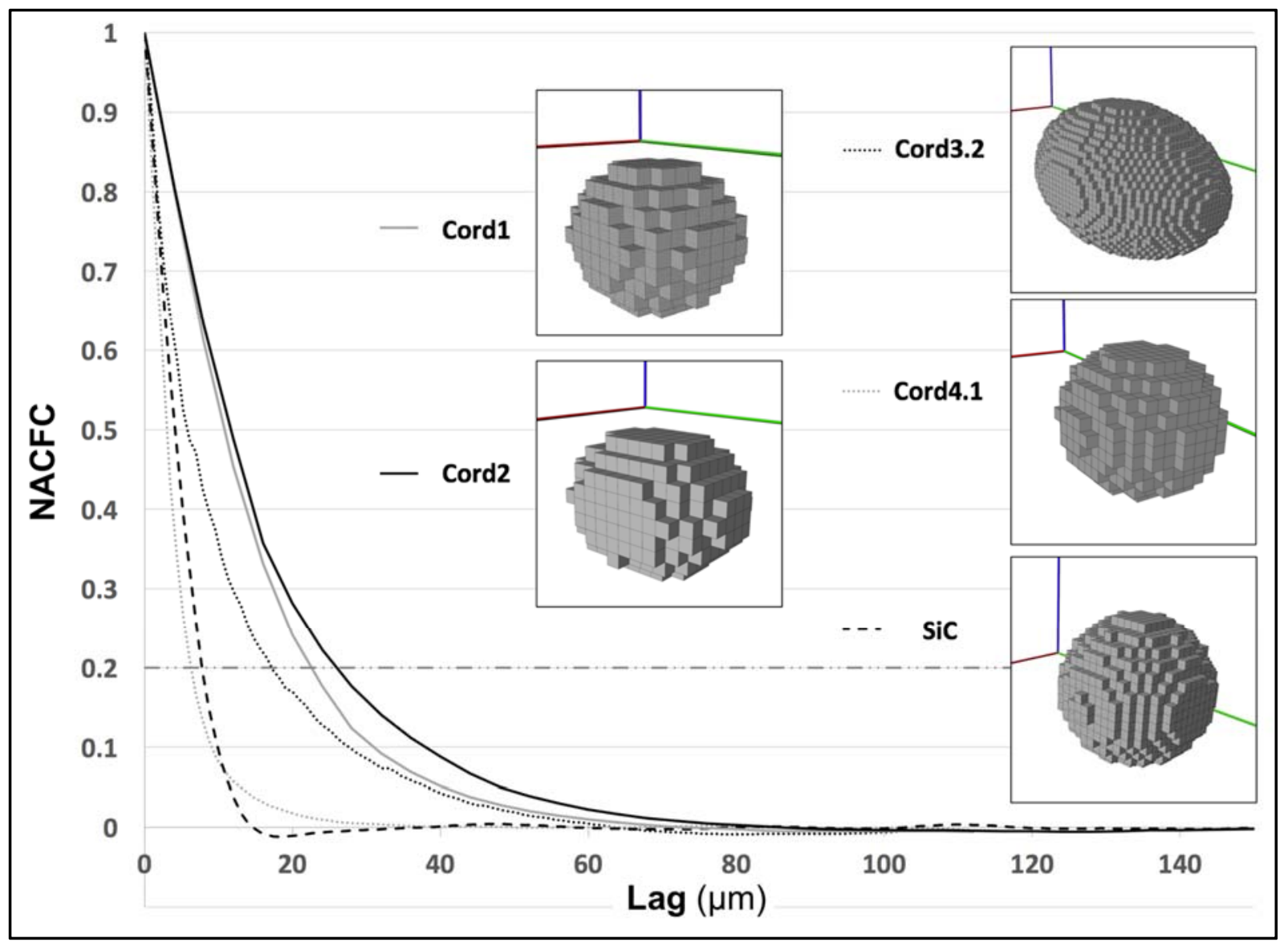

Figure 6: The correlation function $C_{\mathrm{I}}(\mathrm{c})$ along the direction of maximum anisotropy for

five binary sub-volumes as a function of lag c $(\mu \mathrm{m})$. Insets: $3 D$ rendering of the corresponding volumes $V_{I}(0.2)$.

The intersections of the curves NACFC(c) with the horizontal line at 0.2 (dashed line Fig. 6) give, along the directions of maximum anisotropy, the lags for which this level of correlation is reached. Qualitatively, these values are correlated with the size of the main features appearing in the CT sections presented in Fig. 4; small features ( $\mathrm{SiC}$, Cord4) induce small correlation lags and large features (Cord1, Cord2) larger 
correlation lags. This qualitative correlation is mainly due to a scale effect between the different images and cannot be used to estimate the dimensions of any specific features (see Appendix A).

From the 3D rendering of $\mathrm{V}_{\mathrm{I}}(0.2)$ shown in Fig. 6 it is obvious that the degrees of anisotropy are much smaller for these images of real materials than for the synthetic image $\mathrm{I}_{\mathrm{El}}$ (Fig. 2). By definition, the volumes $\mathrm{V}_{\mathrm{I}}(\mathrm{c})$ are discrete objects, and the quality of their discretization varies with the acquisition pixel size and with the size of the features composing the images. Indeed, Cord1, Cord 2 and Cord 3 comprise large features, but for images acquired with $4 \mu \mathrm{m}$ pixel size (Cord1 and Cord2) the discretization quality is much lower than for Cord 3 that was acquired with $0.434 \mu \mathrm{m}$ pixel size (sub-sampled at $0.868 \mu \mathrm{m}$ ). Cord4 and $\mathrm{SiC}$ were also acquired with $0.434 \mu \mathrm{m}$ pixel size (sub-sampled at $0.868 \mu \mathrm{m}$ ), but as they are composed of small features, the discretization quality is only marginally better than for acquisitions with $4 \mu \mathrm{m}$ pixel size.

The parameters through analysis of the volumes $\operatorname{VI}(\mathrm{c})$ for the eight cuboidal binary sub-volumes (Table 1) and the c values between 0.05 and 0.5 were used to estimate the anisotropy in terms of amplitude (DA_Ellips(c)) and orientation. The detailed results are exposed in the following sections.

\section{Permeability computation}

The closure problem (Eq. 4) completed by the boundary conditions (Eq. 5) was solved for each sub-volume extracted from the CT images. This tensor problem reduces to three vector problems, each vector solution corresponding to $\mathbf{D}_{\mathrm{n}}(\mathrm{n}=\mathrm{A}$ or $\mathrm{B}$ or $\mathrm{C})$ a line of the tensor $\mathbf{D}$.

Each vector problem comprises four degrees of freedom: the three components of the line $\mathbf{D}_{\mathrm{n}}$ and the corresponding component of the $\mathbf{d}$ vector. The total number of 
unknown is roughly equal to $\left(4 \cdot \mathrm{NA} \cdot \mathrm{NB} \cdot \mathrm{NC} \cdot \phi_{\mathrm{cr}}\right)$ where $\mathrm{NA}, \mathrm{NB}, \mathrm{NC}$ are the dimensions of the computation domain, and $\phi_{c \tau}$ the porosity fraction of the binary sub-volume (Table 1). The resulting number of unknowns range from $4.810^{7}$ (Cord1) to $1.310^{9}$ (IEl), with an average equal to $4.910^{8}$.

These values clearly indicate that calculations are computationally demanding, resulting in CPU times varying from a few hours to a few days using up to 120 threads on a middle-range Linux server.

Each computed vector field $\mathbf{D}_{\mathrm{n}}(\mathrm{n}=\mathrm{A}$ or $\mathrm{B}$ or $\mathrm{C})$ is related to the local perturbation caused by the solid phase to the macroscopic Darcy flow induced by a macroscopic pressure gradient in the $\mathrm{n}$ direction $[49,50]$. As such, its $3 \mathrm{D}$ representation yields information on the homogeneity of the fluid flow at the sample scale. In Fig. 7 the vector fields are visualised using streamlines and isosurfaces of the module normalised to the maximum component (i.e. $\left.\overline{\mathrm{D}_{\mathrm{n}}}=\frac{\left\|\mathbf{D}_{\mathrm{n}}\right\|}{\operatorname{Max}\left(\left\|\mathbf{D}_{\mathrm{n}}\right\|\right)}\right)$. For all the pixels enclosed by those isosurfaces the corresponding $\overline{D_{n}}$ is larger than the selected threshold (0.35 in Fig. 7). These regions act like bottlenecks and control the permeability, as they correspond to the zones where the fluid is flowing and where the local perturbation is high.

The features shown in Fig. 7 reflect and complement the observations made on the meso-structures of Fig. 4. In cross-sections or CT slices, the size of the features composing the pore space ("pore elements") are discernible, while the connections between them are not. Through the permeability computations both aspects, i.e. "pore elements" and connection sizes, are evidenced, and the consequences of the differences in pore space morphologies are highlighted by these representations. When large "pore elements" are present (Cord1, Cord2, Cord3), the size of the sample is crucial to determine the representativeness of the investigated volume to calculate the 
permeability. For Cord 1 and Cord2 3D images were acquired using a laboratory XCT with pixels of $4.0 \mu \mathrm{m}$. This resulted in a scanned sample much larger than the "pore elements"; consequently, a homogeneous flow distribution in all directions (Fig. 7a and b) is observed. In the $\mathrm{C}$ direction (Fig. 7b) the sample thickness is always limited and, when "pore elements" are large, the two sides of the filter wall might be connected through a few "pore elements" constituting channels that control the global flow.
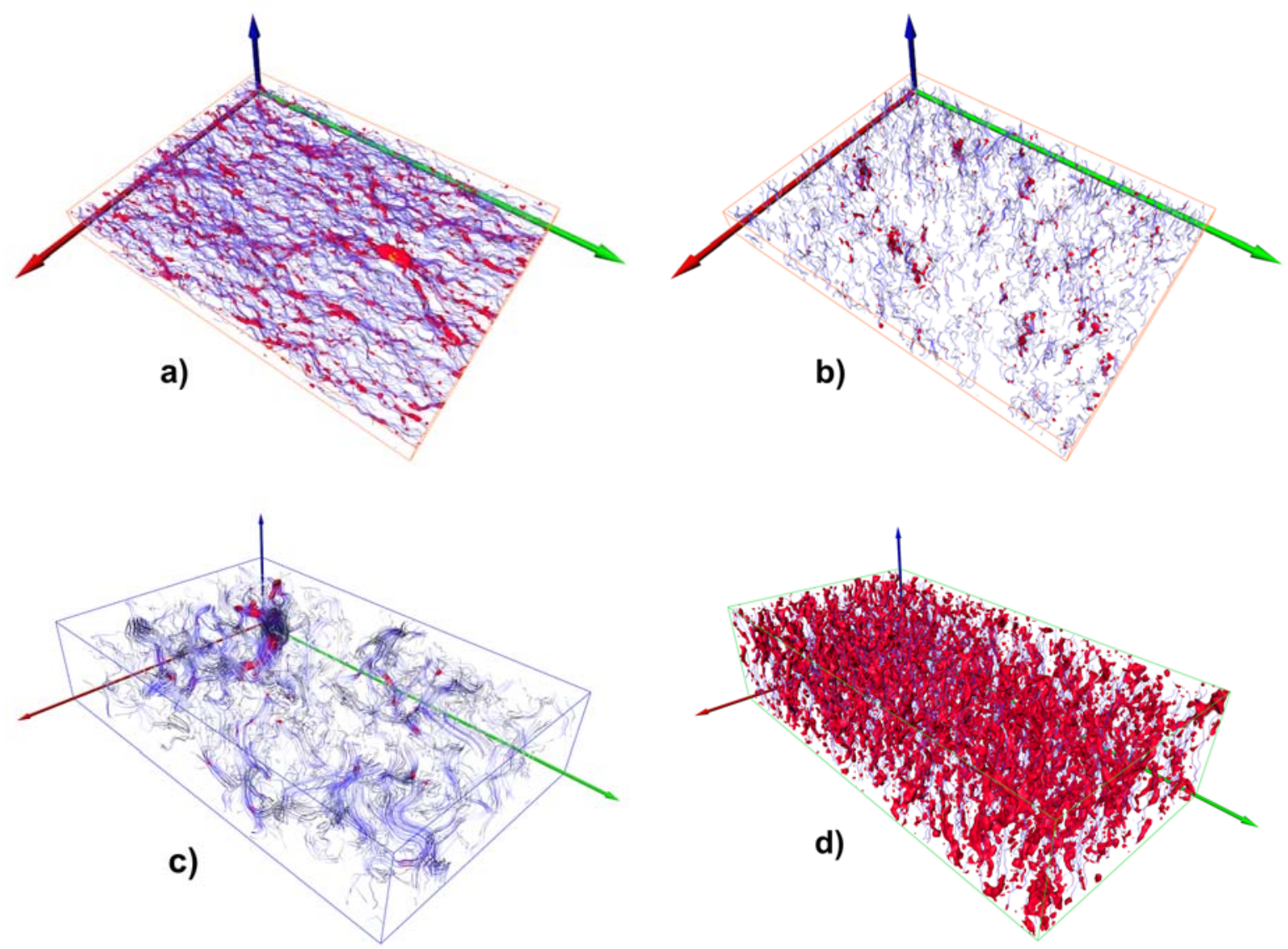

Figure 7: 3D representations of computed vector fields $\boldsymbol{D}_{n}$ as streamlines (in blue) and isosurfaces $\overline{D_{n}}=0.35$ (in red) of the normalized vector modules for Cord1 in the directions $B(\boldsymbol{a})$ and $C(\boldsymbol{b})$, for Cord3.2 in the direction $C(\boldsymbol{c})$, and Cord4.1 in the direction $C(\boldsymbol{d})$.

Homogeneity is then related to the spatial density of these channels (in the A-B plane). Large scanned samples can then be considered as representative (Fig. 7b), whereas the smaller ones, acquired by synchrotron-CT (Cord3, Fig. 7.c) need to be 
more closely inspected, because of the very limited number of channels controlling the flow. The representativeness of high resolution images is good for samples having very small "pore elements", as seen in Fig. 7d, showing $\mathbf{D}_{\mathrm{C}}$ for Cord.4.1. (Note that the vector field for $\mathrm{SiC}$ (not shown in Fig. 7) looks similar to that of Cord4, and is homogeneously distributed).

From the computed vector fields $\mathbf{D}_{\mathrm{n}}(\mathrm{n}=\mathrm{A}, \mathrm{B}, \mathrm{C})$ the permeability tensor of each sample is obtained using Eq. 6. The resulting symmetric full tensor for sample Cord3.1 is given by Eq. 7 .

$$
\mathbf{D}=\left[\begin{array}{ccc}
0.733 & 0.013 & 0.072 \\
0.013 & 0.842 & -0.024 \\
0.072 & -0.024 & 0.639
\end{array}\right]
$$

In this example, as well as for the others, the diagonal terms are dominant, indicating that the axes $(\mathrm{A}, \mathrm{B}, \mathrm{C})$ are not very different from the principal directions. The values of the diagonal terms reported in Table 3 reveal that for all cordierite materials permeability is slightly higher in the extrusion direction B compared to directions A and C. For the synthetic image $\mathrm{I}_{\mathrm{El}}$ these axes are not related to any process, and for $\mathrm{SiC}$, it seems that due to the nature of the material (round nearly monodisperse $\mathrm{SiC}$ sintered powder particles), the extrusion process does not really influence the mesostructure.

The eigenvalues and eigenvectors of the tensors $\mathbf{D}$ can be easily computed, thereby providing a new way to characterise sample anisotropies in amplitude (degree of anisotropy DA) and direction. We define the degree of anisotropy DA_k (k typically symbolises permeability) as the ratio of the larger to the smaller eigenvalues of the permeability tensor. The principal directions of anisotropy correspond to the directions of the eigenvectors of the permeability tensor. For sample Cord3.1, the eigenvalues $\left(\tau_{1}\right.$, $\mathrm{i}=1,3)$ and the eigenvectors $\left(v_{\mathrm{i}}, \mathrm{i}=1,3\right)$ of the tensor given by Eq. 7 are given Table 4 . 
The eigenvalues and eigenvectors for the eight binary sub-volumes (Table

IV.1.2) are listed in Appendix C.

Table 3: Values of the permeability tensor diagonal entries and of the degree of anisotropy calculated by the three methods proposed in the text.

\begin{tabular}{|c||c|c|c|c|c|c|c|c|}
\hline Sample & IEI & Cord1 & Cord2 & Cord3.1 & Cord3.2 & Cord4.1 & Cord4.2 & SiC \\
\hline \hline kAA & 3.21 & 6.01 & 2.45 & 0.73 & 0.85 & 1.91 & 1.92 & 0.70 \\
\hline kBB & 1.53 & 6.70 & 3.31 & 0.84 & 1.04 & 2.12 & 2.13 & 0.65 \\
\hline kcC & 0.52 & 5.93 & 2.64 & 0.64 & 0.76 & 1.80 & 1.81 & 0.63 \\
\hline DA_k & 9.53 & 1.14 & 1.36 & 1.42 & 1.44 & 1.18 & 1.18 & 1.12 \\
\hline DA_Ellips (0.20) & 8.83 & 1.18 & 1.37 & 1.56 & 1.43 & 1.26 & 1.26 & 1.08 \\
\hline DA_Inertia (0.20) & 4.62 & 1.19 & 1.42 & 1.54 & 1.43 & 1.28 & 1.27 & 1.08 \\
\hline
\end{tabular}

Table 4: Eigenvalues and Eigenvectors for sample Cord3.1

\begin{tabular}{|l|c|l|c|c|c|}
\hline \multirow{2}{*}{ Eigenvalues } & \multicolumn{4}{c|}{ Eigenvectors } \\
\cline { 3 - 6 } & & A & B & C \\
\hline$\tau_{1}$ & 0.845 & $v_{1}$ & $4.800 \mathrm{E}-2$ & 0.99400 & $-9.824 \mathrm{E}-2$ \\
\hline$\tau_{2}$ & 0.772 & $v_{2}$ & 0.88500 & $3.282 \mathrm{E}-3$ & 0.46558 \\
\hline$\tau_{3}$ & 0.597 & $v_{3}$ & -0.46312 & 0.10929 & 0.87953 \\
\hline
\end{tabular}

\section{Anisotropy}

As stated above, the permeability tensor can be seen as an implicit function of the mesoscale geometry only. The NACFC is also fully determined by the same meso-scale geometry, and it contains most, if not all, the information about this geometry. This $3 \mathrm{D}$ real-valued function looks much simpler than the local-scale geometry itself (Fig. 2) and the determination of anisotropy is in general possible. We stress that even if it is highly tempting to use it for extracting relevant meso-structural parameters, some limitations need to be taken into account, as presented in Appendix A. From the NACFC we can estimate the meso-structural anisotropy, i.e., in our case, an averaged characteristic of the spatial distribution of the voids and the solid. This property must be distinguished from the functional anisotropies that are related to effective physical properties determined at the global scale. For instance, in the cases of effective mechanical or 
thermal properties of multi-materials, the local-scale geometry must be complemented by the local-scale characteristics of the material (local-scale physical properties) that play an essential role, generally amplifying the difference between structural and functional anisotropies. As both normalised autocorrelation function and permeability tensor are fully determined by the meso-scale geometry, a comparison of the anisotropy characteristics derived from them seems relevant.

For a given binary sub-volume (Table 1), the computed permeability tensor gives one set of eigenvalues and eigenvectors. Equivalent information is obtained from $V_{I}(c)$ for each value of $c$. In this section, anisotropies derived from the permeability tensor and from the properties of $\mathrm{V}_{\mathrm{I}}(\mathrm{c})$ are compared in amplitude (degree of anisotropy DA) and direction (principal direction of anisotropy).

\section{Degree of anisotropy}

Two different estimates of the degree of anisotropy based on the morphology of the volumes $\mathrm{V}_{\mathrm{I}}(\mathrm{c})$ are proposed; DA_Inertia(c), directly derived from the tensor of inertia, and DA_Ellips(c), defined as the ratio of the major to the minor axis of the ellipsoid having the same tensor of inertia (see Appendix B). For the synthetic 3D image IEl these two estimates are very different; DA_Ellips $(0.2)=8.83$ and DA_Inertia $(0.2)=4.62$. DA_k obtained from the permeability tensor computed for this image being 9.53 , DA_Ellips(c) appears to agree much better with DA_k. In the case of all the other samples DA_Ellips(c) and DA_Inertia(c) are very similar (Table 3), indicating that the volumes $\mathrm{VI}_{\mathrm{I}}(\mathrm{c})$ are nearly spherical (see Appendix B). We therefore choose to favour the use of DA_Ellips(c) for the remainder of the paper. 


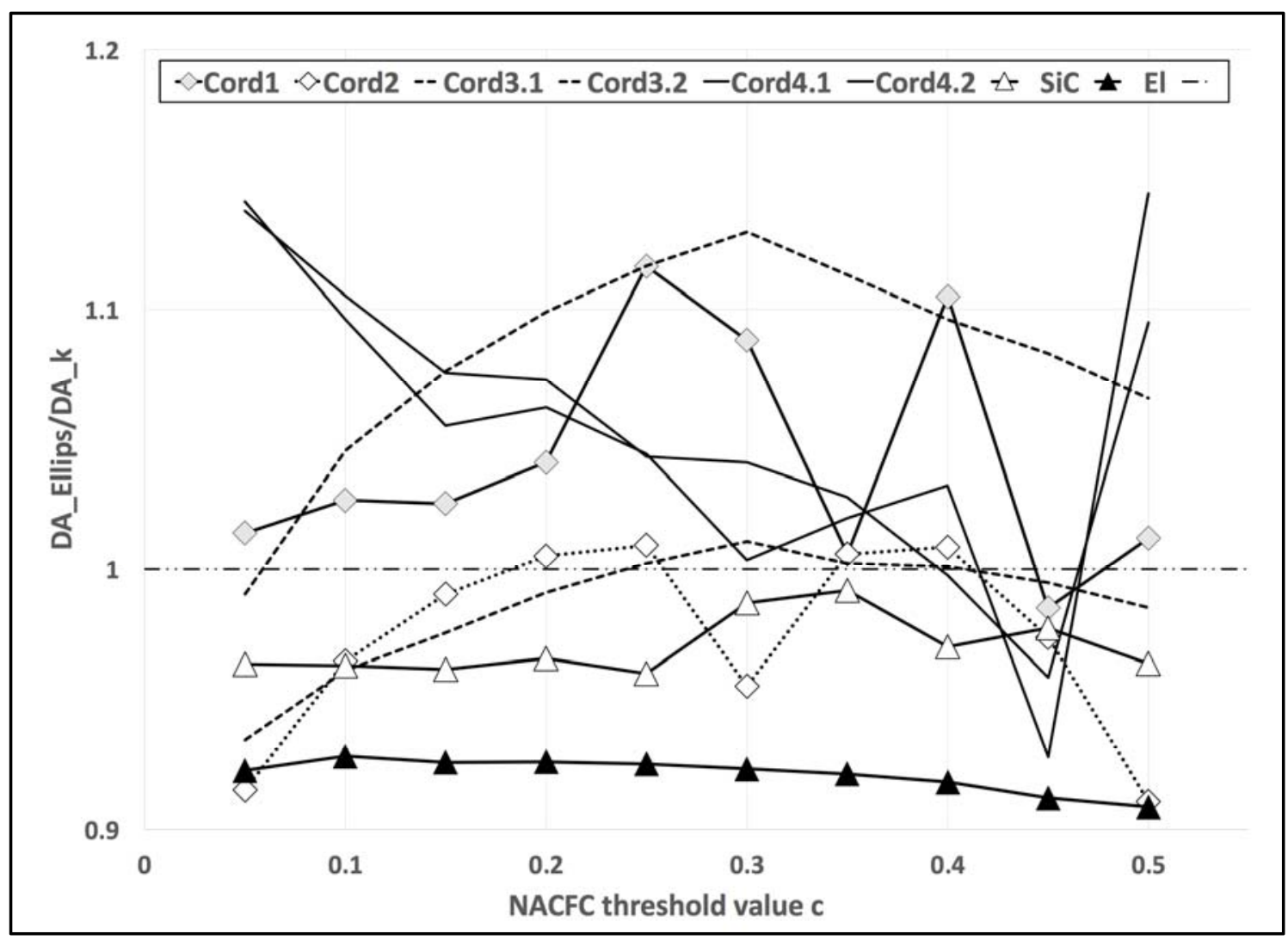

Figure 8: $\overline{D A}$ (the ratio between the anisotropy factors extracted from the NACFC and permeability calculations) as a function of the threshold value c for all the investigated sub-volumes.

The ratio $\overline{\mathrm{DA}}=$ DA_Ellips(c)/DA_k is introduced to evaluate the agreement between the approaches quantifying the meso-structural and the filtration anisotropy. Figure 8 presents the evolutions of $\overline{\mathrm{DA}}$ for all the samples as functions of $\mathrm{c}$, the threshold values used to build $\mathrm{V}_{\mathrm{I}}(\mathrm{c})$.

The main point to be stressed is that all the values of $\overline{\mathrm{DA}}$ lie between 0.9 and 1.15 for $\mathrm{c}$ between 0.05 and 0.5 . This shows that the meso-structural anisotropy (induced by the arrangement of the features composing the 3D images) and the filtration one (permeability) are strongly correlated for this family of materials. It also shows that the NACFC gives a good approximation of the permeability degree of anisotropy at a largely reduced computation cost. 
The evolutions of $\overline{\mathrm{DA}}$ with c can be analysed in more details to extract some specific information relatively to the considered materials. For that, we first distinguish two groups of materials: the materials showing large "pore elements" in Fig. 4, i.e. Cord1, Cord2 and Cord3, and the materials showing small "pore elements" in Fig. 4, i.e. Cord4 and $\mathrm{SiC}$ :

- For the first group, there are two regions limited by a c value between 0.2 and 0.3. Below this value $\overline{\mathrm{DA}}$ is almost constant (Cord1, continuous line with diamonds) or smoothly increasing with c (Cord2, dashed line with diamonds, Cord3, dashed lines). Above this value, smooth (Cord3) or abrupt (Cord1, Cord2) changes appeared. The abrupt variations are directly related to the discretization effect previously described. For Cord1 and Cord 2 the volumes $\mathrm{V}_{\mathrm{I}}(\mathrm{c})$ contain a small number of pixels (Fig.6) and undergo significant changes of shape when $\mathrm{c}$ is varying, inducing the abrupt variations visible in Fig. 9. For Cord3, the variations of $\overline{\mathrm{DA}}$ for the two binary sub-volumes (Cord3.1 and Cord3.2) are nearly parallel with a constant shift, and without noticeable discretization effects. In fact, the sub-volume Cord3.1 is extracted between two crossing regions of the filter web, while Cord3.2 is extracted across one of these crossing regions. Consequently, Cord3.2 is more homogeneous and isotropic than Cord3.1 (see Fig. 9a).

- For the second group, variations of $\overline{\mathrm{DA}}$ with $\mathrm{c}$ are of two kinds: a smooth decrease for $\mathrm{c} \leq 0.3$ followed by abrupt variations in the case of Cord 4 (continuous lines), an almost constant value for $\mathrm{c} \leq 0.25$, followed by variations of limited amplitude in the case of $\mathrm{SiC}$ (continuous line with triangles). These images are affected by the discretization procedures, because the pixel size is small $(0.434 \mu \mathrm{m}$, sub-sampled at $0.868 \mu \mathrm{m})$, but "pore elements" are also small. 
For Cord4, the variations of $\overline{\mathrm{DA}}$ for the two binary sub-volumes are very similar.

This can be explained by the facts that the pore space meso-structure is

homogeneous (and therefore all sub-volumes tend to have similar geometric anisotropy), and the two volumes are extracted at similar distances from the crossing points of the filter web (see Fig. 9b).
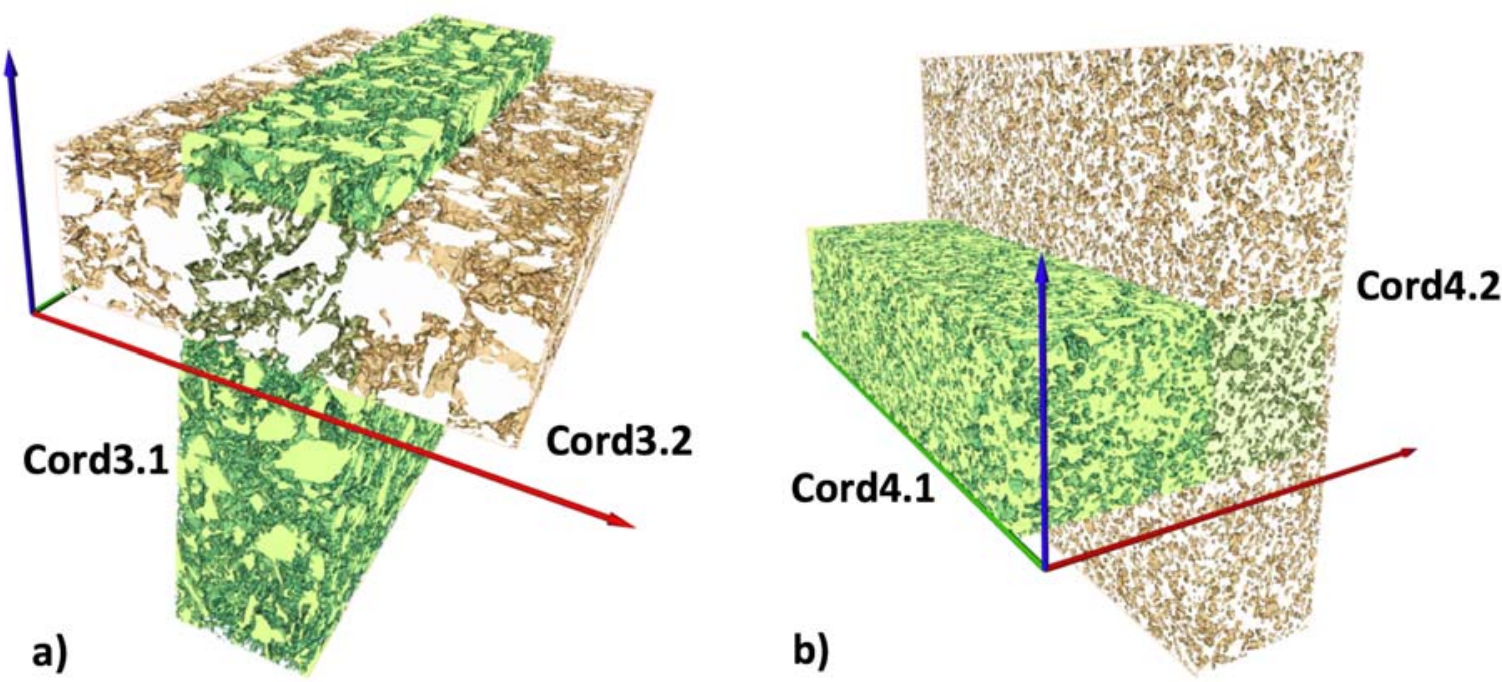

Figure 9: (a) Location of Cord3.1 and Cord3.2 sub-volumes; (b) Location of Cord4.1 and Cord4.2 sub-volumes.

- For the synthetic image $\mathrm{I}_{\mathrm{El}}, \overline{\mathrm{DA}}$ is essentially constant, and, for this very homogeneous image, the value of $\overline{\mathrm{DA}}$ effectively quantifies the relation between the meso-structural and the filtration estimates of anisotropy.

From the comments presented above, it appears that, if a single value has to be selected for $\mathrm{c}, \mathrm{c}$ equal to 0.2 is a good choice. Therefore, this value will be used for DA in the rest of this work (see Table 3).

The NACFC also contains information about the scale, i.e. a given degree of correlation c is reached at a given lag in a specific direction, and DA_Ellips(c) shows the differences in lag according to direction (Fig. 3). The decrease of $\overline{\mathrm{DA}}$ towards 1 with 
increasing $\mathrm{c}$ for Cord 4 can then be interpreted, for that material, as an increase of the correlation between meso-structural and filtration anisotropies with an optimum at $\mathrm{c}=0.3$. This corresponds to a lag of about $16 \mu \mathrm{m}$ in one direction (Fig. 3), and $13.5 \mu \mathrm{m}$ in the other $(\overline{\mathrm{DA}}=1$ implying that DA_Ellips $=$ DA_k $=1.18$, Table 3$)$. These values seem consistent with the meso-structure presented Fig. $4(16 \mu \mathrm{m}=1 / 19$ of the side of the section). Nevertheless, this interpretation cannot be generalised, as for IEL and $\mathrm{SiC}$ the correlation between the two types of anisotropy is independent of c, and consequently of the explored scales. The prominent results of this section are that, for these materials, meso-structural anisotropy and filtration anisotropy are clearly correlated. On a case by case basis, some information about the structure can be extracted from the small variations of $\overline{\mathrm{DA}}$ with $\mathrm{c}$.

\section{Directions of anisotropy}

The second characteristic after the degree of anisotropy is the direction of anisotropy. For the cordierite samples the main direction of anisotropy obtained from the permeability tensor is close to the direction of extrusion B (Appendix C). To compare these directions with the ones corresponding to the principal directions given by analysing the morphology of $V_{I}(c)$, the graphic presented Fig. 10 is used. It shows the projection on the plane $(\mathrm{A}, \mathrm{C})$ of the surface of a unit sphere with centre at the origin of the coordinate system $(\mathrm{A}, \mathrm{B}, \mathrm{C})$. All points represent the intersections of the principal direction lines (drawn through the origin) and the unit sphere. The angle between a given line and the $\mathrm{B}$ axis is equal to 90 degrees minus the latitude of the intersection point on the surface of the sphere (circles corresponding to some values of latitude are drawn in light grey). For sample Cord3, points corresponding to permeability (triangles) or NACFC (squares) are confined in a limited zone (Fig. 10 central image) for both of 
the binary sub-samples (Cord3.1 in white, Cord3.2 in grey).

Focussing on the central region of Fig. 10 (upper inset), it appears that the intersection points of the main direction of $\mathrm{V}_{\mathrm{Cord} 3.1}(\mathrm{c})$ and $\mathrm{V}_{\mathrm{Cord} 3.2}(\mathrm{c})$ tend towards the intersection points of the principal direction of the permeability tensor (triangles) when c increases.

On the lower inset of Fig. 10 the pairs of intersection points corresponding to the main direction of $\mathrm{V}_{\mathrm{I}}(0.2)$ and of the permeability principal direction (symbols with a black dots) are displayed for each of the six cordierite binary sub-samples. All these directions have a maximum misalignment of 10 degrees with the axis B.

Table 5: Components of the principal directions of the permeability tensor and of the volume $V_{I}(0.2)$ for the synthetic and SiC samples.

\begin{tabular}{|c||c|c|c|c|c|c||}
\hline \multicolumn{1}{|c||}{ Sample } & \multicolumn{3}{c|}{ IEI $_{\text {SiC }}$} \\
\hline Axes & A & B & C & A & B & C \\
\hline \hline Permeability & 0.99998 & -0.00276 & -0.00450 & 0.97144 & 0.22907 & -0.06196 \\
\hline $\mathbf{V}_{\mathbf{I}(\mathbf{0 . 2})}$ & 0.99997 & 0.00044 & 0.00047 & 0.84866 & 0.50899 & -0.14393 \\
\hline
\end{tabular}

As seen above, for all the cordierite samples the main direction of anisotropy is strongly correlated with the direction of extrusion $\mathrm{B}$. This is not the case for the synthetic image IEL, for which this notion is irrelevant, and for SiC, for which the mesostructure appears as unaffected by the extrusion process. The main directions of permeability and $\mathrm{V}_{\mathrm{I}}(0.2)$ are reported in Table 5 for the synthetic image and $\mathrm{SiC}$ showing for them also a strong correlation between filtration and meso-structural anisotropy. 


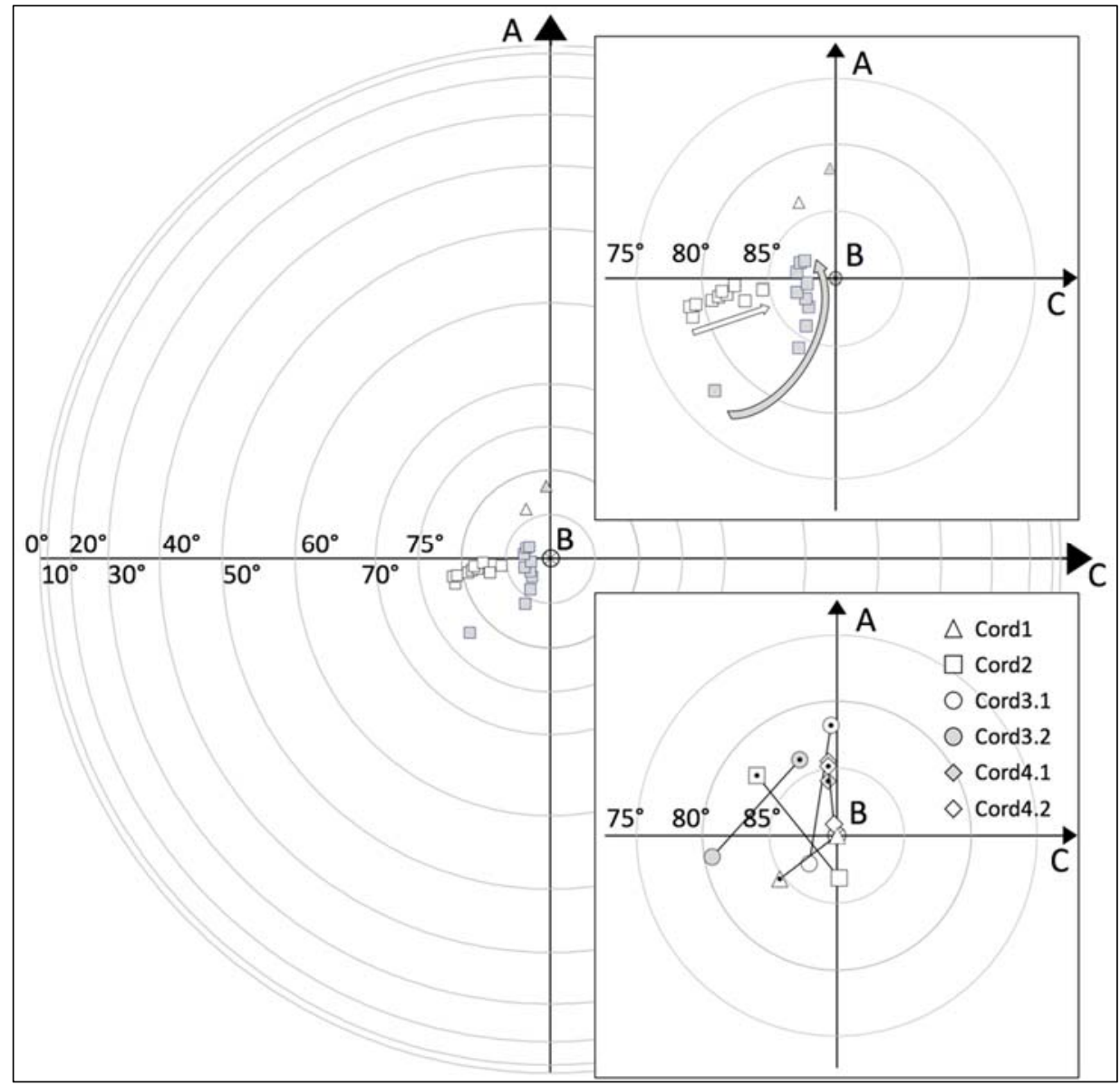

Figure 10: Intersections between the principal directions of anisotropy (calculated from the NACFC at various values of $c$, and drawn passing through the origin of the sphere) and the unit sphere. On the upper right corner, a magnification of the central region is shown, with the evolution of the intersection point for increasing values of $c$. In the lower right corner, the intersections of the principal direction of the permeability tensor (dotted symbols) and of the principal direction of $V_{\mathrm{I}}(0.2)$ with the unit sphere are compared (linked by a line for the same sample).

\section{Discussion}

The results presented above indicate a strong correlation between the meso-structural anisotropy DA_Ellips(0.2) estimated using the NACFC and the filtration anisotropy DA_k estimated from the permeability tensor. Extending this correlation to any 
functional anisotropy would be misleading, as in many cases local-scale physical properties also influence the results. Even in the cases where the only local parameter is the local geometry, different modes of transfer might induce different degrees of anisotropy as shown by Sato et al. [53] for Berea sandstone on samples having a layered structure that induced a permeability anisotropy of 8.5 and a diffusion anisotropy of 1.4. This difference is a consequence of the form of the closure problems (Eq. 4 and 5 for filtration) that determines the effects of local-geometry on effective properties.

This work focused on NACFC vs. permeability and logically all results were generated using binary images (solid/pore space) as required for permeability computations. It is of importance to notice that NACFC can be computed on the greylevel images as easily as on the binarised ones. The direct use of grey-level images would circumvent the critical segmentation step, which inevitably influences the permeability computation.

To assess the consequences of using a grey-level image instead of a segmented one, the following procedure was followed:

- A sub-volume of $600 \times 600 \times 540$ pixels was extracted from the centre of the original grey-level image of Cord4 (with a pixel size of $0.434 \mu \mathrm{m}$ ).

- This sub-volume was then sub-sampled by factors of $2,3,4,5,6,8$, and 9 .

- For each of these sub-volumes, the NACFC was calculated, and $\overline{\mathrm{DA}}^{*}(0.2)$ evaluated from the corresponding volumes $\mathrm{V}_{\mathrm{I}}(0.2)$. For a given pixel size, $\overline{\mathrm{DA}}^{*}(0.2)$ is the ratio of DA_Ellips $(0.2)$ of $\mathrm{V}_{\mathrm{I}}(0.2)$ (for the corresponding subvolume) to DA_k given in Table 3.

Figure 11 shows a section of the high resolution sub-volume (a), and approximately the same section sub-sampled by a factor of 8 (b). The representation of 
the local meso-geometry is of course strongly altered. The consequences for the anisotropy quantification are shown in Fig. 11c (showing the evolution of $\overline{\mathrm{DA}}^{*}(0.2)$ with pixel size). The dashed line indicates the value of $\overline{\mathrm{DA}}(0.2)$ for Cord4 previously calculated. It appears that globally, the approximation of DA_k obtained from the NACFC of the sub-sampled sub-volumes (DA_Ellips $(0.2)$ of $V_{I}(0.2)$ ) is acceptable for sub-sampling factors between 1 and 8 (pixel sizes between $0.434 \mu \mathrm{m}$ and $3.47 \mu \mathrm{m}$ ). The two first points $(0.434 \mu \mathrm{m}$ and $0.868 \mu \mathrm{m})$ are very close to the dashed line. This confirms that Cord4 is homogeneous, as previously indicated, and that the first subsampling does not alter the features of the local meso-geometry. These results denote that a good approximation of the filtration anisotropy can be achieved by calculating the NACFC of a grey-level 3D image even if the local meso-geometry is poorly resolved.

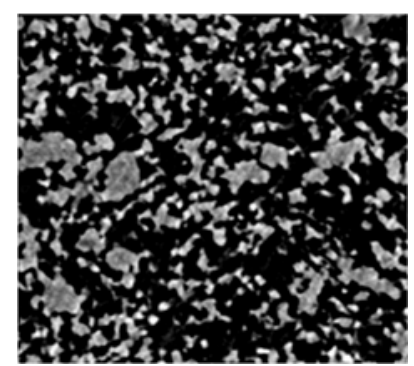

a)

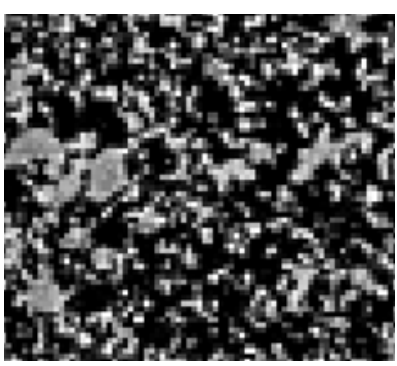

b)

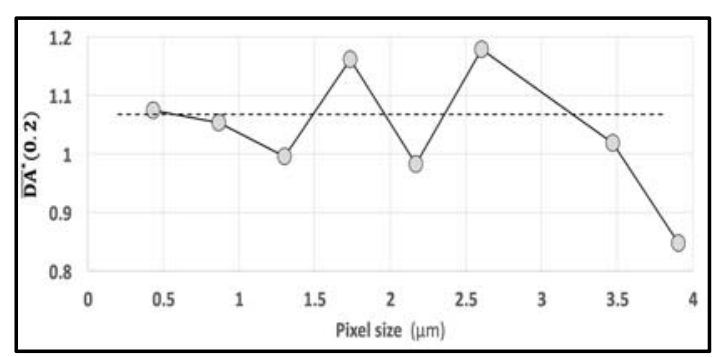

c)

Figure 11: The same section of Cord4 (260 x $\left.234 \mu \mathrm{m}^{2}\right)$ with pixels of $0.434 \mu \mathrm{m}$ (a) and 3.47 $\mu \mathrm{m}$ (b). Evolution of $\overline{D A}^{*}(0.2)$ with the pixel size (c). The dashed line indicates the value obtained for the binary image.

The permeability and the autocorrelation functions yield relevant information about the anisotropy of their filtration and morphological properties. For cordierite samples, the direction of extrusion is clearly the principal direction of anisotropy. As found in $[54,55]$, the degree of anisotropy of the meso-structure is similar to that of the crystal texture, which in turn induces anisotropy of the coefficient of thermal expansion and Young's modulus (since the properties themselves are anisotropic). This correlation 
is strongly influenced by micro-cracking in DPF materials [56]. In the present case, however, pore space is dominant over micro-cracking (see also the analysis in $[29,30]$ ), because microcracks have negligible dimensions. In fact, Table IV.4.1 shows that an increase in meso-structural anisotropy (compare Cord 1 to Cord 2 or Cord3) corresponds to an increase in permeability anisotropy. It is interesting to note that for $\mathrm{SiC}$ the meso-structure is almost isotropic (DA_Ellips $(0.2)=1.08)$, but DA_Ellips(0.2)/DA_k $=0.96$, which implies that the filtration properties of $\mathrm{SiC}$ are more textured than its physical and mechanical properties [57]. It is generally confirmed that cordierite materials possess relatively weak morphological texture, and that increasing porosity fraction (and decreasing the size of the pore space features, i.e. smaller but more pore space features) actually decreases the degree of anisotropy. In fact, the thermal and physical properties also become less anisotropic [58].

\section{Conclusions}

To study quantitatively the anisotropy of both meso-structure and fluid flow in Diesel Particulate Filter (DPF) materials we used the normalised autocorrelation function of the centred image (NACFC), and permeability calculations for 3D CT data reconstructions.

For large 3D images such as the ones considered here, permeability calculations are computationally demanding, therefore requiring advanced numerical tools. From the resulting permeability tensor, the principal direction and the degree of anisotropy are easily deduced.

On the contrary, the NACFC is easy to calculate, but the resulting $3 \mathrm{D}$ realvalued function is difficult to interpret. For that, we first clarified, by a theoretical analysis, the nature of the information that can be extracted (Appendix A), and we developed a new method to quantify the anisotropy based on the morphological 
properties of the volume $\mathrm{V}_{\mathrm{I}}(\mathrm{c})$ constituted by all the pixels for which NACFC is larger than a certain threshold $\mathrm{c}$. Examining the variations of these properties for $\mathrm{c}$ varying between 0.05 and 0.5 , we shown that, for all the considered samples, the values provided by both approaches are in very good agreement. If a single value of $\mathrm{c}$ has to be used, c equal 0.2 is a good choice.

We focused our attention on Cordierite and $\mathrm{SiC}$, two widely used materials for DPF. In general, meso-structural anisotropy directly affects physical properties, but the two may only be loosely linked. In this work we found that meso-structural and filtration anisotropies are strongly correlated for both the degree of anisotropy and the orientation. In line with the few other studies available in the open literature, it appears that cordierite materials are weakly anisotropic and clearly influenced by the extrusion process that determines the main direction of anisotropy. A slight dependence of anisotropy on the feature size of the meso-structure as seen on 2D images was put into evidence for a family of cordierite materials (all having porosity fraction around 0.5 ): the finer the grain and the "pore elements" size, the more anisotropic the meso-structure and the permeability. Since the meso-structure in such materials is dictated by the raw powder and by the pore-former particle size, we deduce that controlling those parameters would allow controlling the degree of anisotropy of both the filtration and of the physical properties of such materials.

The autocorrelation function can yield several insights on the meso-structure, such as the dependence of the degree of orientation on the length range of the mesostructure considered in the analysis: homogeneous structures such as $\mathrm{SiC}$ (and ideal materials) show the same degree of anisotropy at all length scales.

The approach based on the analysis of the NACFC that we proposed appears as precise and efficient even for materials having a small degree of anisotropy. Finally, we 
showed that the NACFC applied on grey-level images can give a good estimate of the degree of anisotropy even with limited resolution.

\section{Acknowledgments}

DB acknowledges financial support from BAM, in the frame of the 'Guest

Scientists' programme.

The authors acknowledge the anonymous reviewer for his/her valuable work.

\section{Disclosure statement}

The authors have no competing interests.

\section{Data availability statement}

Data used in this work are available from the corresponding author upon reasonable request and with the permissions of BAM and CNRS.

\section{References}

[1] B. Sander, An introduction to the study of fabrics of geological bodies, Pergamon, Oxford, 1970, p. 641

[2] A.M. Gokhale, Quantitative Characterization and Representation of Global Microstructural Geometry, in ASM Handbook Volume 9: Metallography and Microstructures, ed. by G.V. Voort (ASM International, Materials Park, 2004)

[3] A.M. Parfitt, M.K. Drezner, F.H. Glorieux, J.A. Kanis, H. Malluche, P.J. Meunier, S.M. Ott, and R.R. Recker, Bone histomorphometry: Standardization of nomenclature, symbols, and units: Report of the asbmr histomorphometry nomenclature committee, Journal of Bone and Mineral Research 2 (1987), pp. 595-610.

[4] W.J. Whitehouse, The quantitative morphology of anisotropic trabecular bone, Journal of Microscopy 101 (1974), pp. 153-168.

[5] M.Y.M. Chiang, X. Wang, F.A. Landis, J. Dunkers, and C.R. Snyder, Quantifying the directional parameter of structural anisotropy in porous media, Tissue Engineering 12 (2006), pp. 1597-1606.

[6] P. Varga, and P.K. Zysset, Sampling sphere orientation distribution: An efficient method to quantify trabecular bone fabric on grayscale images, Medical Image Analysis 13 (2009), pp. 530-541. 
[7] A. Odgaard, E.B. Jensen, and H.J.G. Gundersen, Estimation of structural anisotropy based on volume orientation. A new concept, Journal of Microscopy 157 (1990), pp. 149-162.

[8] M.Y.M. Chiang, F.A. Landis, X. Wang, J.R. Smith, M.T. Cicerone, J. Dunkers, and Y. Luo, Local thickness and anisotropy approaches to characterize pore size distribution of three-dimensional porous networks, Tissue Engineering - Part C: Methods 15 (2009), pp. 65-76.

[9] L.M. Cruz-Orive, L.M. Karlsson, S.o. Larsen, ren E., and F. Wainschtein, Characterizing anisotropy: A new concept, Micron and Microscopica Acta 23 (1992), pp. 75 - 76.

[10] A. Odgaard, J. Kabel, B. van Rietbergen, M. Dalstra, and R. Huiskes, Fabric and elastic principal directions of cancellous bone are closely related, Journal of Biomechanics 30 (1997), pp. 487 - 495.

[11] J. Millard, P. Augat, T.M. Link, M. Kothari, D.C. Newitt, H.K. Genant, and S. Majumdar, Power spectral analysis of vertebral trabecular bone structure from radiographs: Orientation dependence and correlation with bone mineral density and mechanical properties, Calcified Tissue International 63 (1998), pp. 482-489.

[12] Y. Xiang, V.R. Yingling, R. Malique, C. Yang Li, M.B. Schaffler, and T. Raphan, Comparative assessment of bone mass and structure using texture-based and histomorphometric analyses, Bone 40 (2007), pp. 544-552.

[13] P.K. Saha, and F.W. Wehrli, A robust method for measuring trabecular bone orientation anisotropy at in vivo resolution using tensor scale, Pattern Recognition 37 (2004), pp. 1935-1944.

[14] M. Rotter, A. Berg, H. Langenberger, S. Grampp, H. Imhof, and E. Moser, Autocorrelation analysis of bone structure, Journal of Magnetic Resonance Imaging 14 (2001), pp. 87-93.

[15] M. Wald, B. Vasilic, P.K. Saha, and F.W. Wehrli, Study of trabecular bone microstructure using spatial autocorrelation analysis, Proceedings of the SPIE, Volume 5746 (2005), pp. 291-302.

[16] M. Faessel and D. Jeulin, Segmentation of 3D microtomographic images of granular materials with the stochastic watershed, Journal of Microscopy, Vol. 239 (2010), pp. 17-31.

[17] Y. Jiao, F. H. Stillinger and S. Torquato, Modeling heterogeneous materials via two-point correlation functions: Basic principles, Physical Review E, 76 (2007), 031110 .

[18] F.W. Wehrli, H. Kwon Song, P.K. Saha, and A.C. Wright, Quantitative MRI for the assessment of bone structure and function, NMR in biomedicine Special Issue: "NMR of the Musculoskeletal System" (2006), pp. 731-764.

[19] M.J. Wald, B. Vasilic, P.K. Saha, and F.W. Wehrli, Spatial autocorrelation and mean intercept length analysis of trabecular bone anisotropy applied to in vivo magnetic resonance imaging, Medical Physics 34 (2007), pp. 1110-1120.

[20] J. Adler, Ceramic diesel particulate filters, International Journal of Applied Ceramic Technology 2 (2005), pp. 429-439.

[21] G. Bruno, A.M. Efremov, C. An, and S. Nickerson, Not All Microcracks are Born Equal: Thermal vs. Mechanical Microcracking in Porous Ceramics, Advances in Bioceramics and Porous Ceramics IV: Ceramic Engineering and Science Proceedings 32 (2011).

[22] Y. Kasai, K. Kumazawa, and W. Kotani, Cordierite ceramic filter and method of producing the same, E. P. Office ed., 1994. 
[23] D. Munroe Beall, G.A. Merkel, and M.J. Murtagh, Cordierite porous ceramic honeycomb articles with delayed microcrack evolution, U. S. Patent ed., Corning Incorporated, USA, 2015.

[24] C. Bubeck, Direction dependent mechanical properties of extruded cordierite honeycombs, Journal of the European Ceramic Society 29 (2009), pp. 3113-3119.

[25] G. Bruno, A.M. Efremov, B. Clausen, A.M. Balagurov, V.N. Simkin, B.R. Wheaton, J.E. Webb, and D.W. Brown, On the stress-free lattice expansion of porous cordierite, Acta Materialia 58 (2010), pp. 1994-2003.

[26] A. Shyam, E. Lara-Curzio, A. Pandey, T.R. Watkins, K.L. More, and T. Ohji, The Thermal Expansion, Elastic and Fracture Properties of Porous Cordierite at Elevated Temperatures, Journal of the American Ceramic Society 95 (2012), pp. 1682-1691.

[27] T. Gordon, A. Shyam, and E. Lara-Curzio, The Relationship Between Microstructure and Fracture Toughness for Fibrous Materials for Diesel Particulate Filters, Journal of the American Ceramic Society 93 (2010), pp. 11201126.

[28] A. Lichtner, D. Roussel, D. Jauffrès, C.L. Martin, R.K. Bordia, and G. Franks, Effect of Macropore Anisotropy on the Mechanical Response of Hierarchically Porous Ceramics, 99 (2016).

[29] A. Kupsch, A. Lange, M.P. Hentschel, Y. Onel, T. Wolk, A. Staude, K. Ehrig, B.R. Müller, and G. Bruno, Evaluating Porosity in Cordierite Diesel Particulate Filter Materials, Part 1- X-ray Refraction, Journal of Ceramic Science and Technology 04 (2013), pp. 196-176.

[30] Y. Onel, A. Lange, A. Staude, K. Ehrig, A. Kupsch, M.P. Hentschel, T. Wolk, B.R. Müller, and G. Bruno, Evaluating Porosity in Cordierite Diesel Particulate Filter Materials, Part 2 Statistical Analysis of Computed Tomography Data, Journal of Ceramic Science and Technology 05 (2013), pp. 13-22.

[31] A. Kupsch, B.R. Müller, A. Lange, and G. Bruno, Microstructure characterisation of ceramics via $2 D$ and $3 D X$-ray refraction techniques, Journal of the European Ceramic Society 37 (2017), pp. 1879-1889.

[32] S.-H. Leigh, and C.C. Berndt, Modelling of elastic constants of plasma spray deposits with ellipsoid-shaped voids, Acta Materialia 47 (1999), pp. 1575-1586.

[33] A.P. Roberts, and E.J. Garboczi, Elastic properties of model porous ceramics, Journal of American Ceramic Society 83 (2000), pp. 3041-48.

[34] K. Schmidt, and J. Becker, Generating 3D model of microporous ceramics, Advanced Engineering Materials 15 (2013), pp. 40-45.

[35] T.P. de Carvalho, H.P. Morvan, D.M. Hargreaves, H. Oun, and A.J.T.i.P.M. Kennedy, Pore-Scale Numerical Investigation of Pressure Drop Behaviour Across Open-Cell Metal Foams, 117 (2017), pp. 311-336.

[36] M. Masoudi, A. Heibel, and P.M. Then, Predicting Pressure Drop of Wall-Flow Diesel Particulate Filters - Theory and Experiment, 2000.

[37] W. Görner, M.P. Hentschel, B.R. Müller, H. Riesemeier, M. Krumrey, G. Ulm, W. Diete, U. Klein, and R. Frahm, BAMline: the first hard X-ray beamline at BESSY II, Nuclear Instruments and Methods in Physics Research Section A: Accelerators, Spectrometers, Detectors and Associated Equipment 467-468 (2001), pp. 703-706.

[38] A. Rack, S. Zabler, B.R. Müller, H. Riesemeier, G. Weidemann, A. Lange, J. Goebbels, M. Hentschel, and W. Görner, High resolution synchrotron-based radiography and tomography using hard X-rays at the BAMline (BESSY II), 
Nuclear Instruments and Methods in Physics Research Section A: Accelerators, Spectrometers, Detectors and Associated Equipment 586 (2008), pp. 327-344.

[39] A. Lange, M.P. Hentschel, A. kupsch, and B.R. Müller, Numerical correction of $X$-ray detector backlighting, International Journal of Materials Research 103 (2012).

[40] A. Kupsch, M.P. Hentschel, A. Lange, and B.R. Müller, Einfaches Korrigieren des Hinterleuchtens von Röntgendetektoren, Materials Testing 55 (2013).

[41] C.J. Thissen, and M.T. Brandon, An autocorrelation method for threedimensional strain analysis, Journal of Structural Geology 81 (2015), pp. 135154.

[42] D. Bernard, O. Guillon, N. Combaret, and E. Plougonven, Constrained sintering of glass films: Microstructure evolution assessed through synchrotron computed microtomography, Acta Materialia 59 (2011), pp. 6228-6238.

[43] R.P. Heilbronner, The autocorrelation function: an image processing tool for fabric analysis Tectonophysics 212 (1992), pp. 351-370.

[44] A. Z. Zinchenko, Algorithm for random close packing of spheres with periodic boundary conditions, Journal of Computational Physics, 114 (1994), pp. 298-307.

[45] R. Heilbronner, and S. Barrett, Image Analysis in Earth Sciences. Microstructures and Textures of Earth Materials, Springer-Verlag Berlin, 2014.

[46] M. Frigo, and S.G. Johnson, The Design and Implementation of FFTW3, Proceedings of the IEEE 93 (2005), pp. 216-231.

[47] H. Darcy, Les fontaines publiques de la ville de Dijon, Victor Dalmont, Libraire des Corps imperiaux des ponts et chaussées et des mines, 1856.

[48] S. Whitaker, The Method of Volume Averaging, Theory and Applications of Transport in Porous Media, 1999.

[49] D. Bernard, Ø. Nielsen, L. Salvo, and P. Cloetens, Permeability assessment by 3D interdendritic flow simulations on microtomography mappings of Al-Cu alloys, Materials Science and Engineering: A 392 (2005), pp. 112-120.

[50] D. Bernard, Using the volume averaging technique to perform the first change of scale for natural random porous media, in Advanced Methods for Groundwater Pollution Control, G. Gambolati and G. Verri eds., Springer Vienna, Vienna, 1995, pp. 9-24.

[51] F. Xiao, and X. Yin, Geometry models of porous media based on Voronoi tessellations and their porosity-permeability relations, Computers \& Mathematics with Applications 72 (2016), pp. 328-348.

[52] J. Hommel, E. Coltman, and H. Class, Porosity-Permeability Relations for Evolving Pore Space: A Review with a Focus on (Bio-)geochemically Altered Porous Media, Transport in Porous Media 124 (2018), pp. 589-629.

[53] M. Sato, K. Panaghi, N. Takadai and M. Takeda, Effect of Bedding Planes on the Permeability and Diffusivity Anisotropies of Berea Sandstone, Transport in Porous Media 127 (2019), pp. 587-603.

[54] G. Bruno, and S. Vogel, Calculation of the Average Coefficient of Thermal Expansion in Oriented Cordierite Polycrystals, Journal of the American Ceramic Society 91 (2008), pp. 2646-2652.

[55] G. Bruno, and S.C. Vogel, Simultaneous determination of high-temperature crystal structure and texture of synthetic porous cordierite, Journal of Applied Crystallography 50 (2017), pp. 749-762.

[56] G. Bruno, A.M. Efremov, B.R. Wheaton, and J.E. Webb, Microcrack orientation in porous aluminum titanate, Acta Materialia 58 (2010), pp. 6649-6655. 
[57] G. Bruno, A.M. Efremov, A.N. Levandovskiy, I. Pozdnyakova, D.J. Hughes, and B. Clausen, Thermal and Mechanical Response of Industrial Porous Ceramics, Materials Science Forum 652 (2010), pp. 191-196.

[58] G. Bruno, A.M. Efremov, C.P. An, B.R. Wheaton, and D.J. Hughes, Connecting the macro and microstrain responses in technical porous ceramics. Part II: microcracking, Journal of Materials Science 47 (2012), pp. 3674-3689.

[59] C. Lin, Microgeometry I: Autocorrelation and rock microstructure, Journal of the International Association for Mathematical Geology 14 (1982), pp. 343-360.

\section{Appendix A: Why NACFC $(v)$ of an image cannot be used for grain size estimation}

Attempts to characterize rock meso-structure using $\mathrm{CI}_{\mathrm{I}}(\mathbf{v})$, the $\mathrm{NACFC}(\mathbf{v})$ of image $\mathrm{I}$, as defined by Eq. 2, are already dated $[43,59]$, but the transition to quantification of grains sizes, even proposed as modules in some software [45], generally leads to poor approximations for dense aggregates [41]. The main reason is linked to a property of the function $\mathrm{C}_{\mathrm{I}}(\mathbf{v})$ that we expose in this appendix.

First we must define what is the inversed image $\operatorname{Inv}(\mathbf{u})$ of a real valued image $\mathrm{I}(\mathbf{u})$ :

$$
\mathrm{I}_{\mathrm{Inv}}(\mathbf{u})=-\mathrm{I}(\mathbf{u})+\operatorname{Max}[\mathrm{I}(\mathbf{u})]+\operatorname{Min}[\mathrm{I}(\mathbf{u})]
$$

If $\mathrm{I}(\mathbf{u})$ is a binary image $(0 / 1), \operatorname{Inv}(\mathbf{u})$ is also binary, with the pixels with grey value $=0$ corresponding to the pixels of $\mathrm{I}(\mathbf{u})$ with grey value $=1$, and vice-versa. The background of $\mathrm{I}(\mathbf{u})$ is now the object of $\operatorname{Inv}(\mathbf{u})$ and vice versa.

Using the definition of the autocorrelation function given by Eq. 1, taking into account the linearity of the averaging operator $<>$ and the fact that the average of a constant is equal to this constant, the following relation between the ACFs of $\mathrm{I}(\mathbf{u})$ and $\mathrm{I}_{\mathrm{Inv}}(\mathbf{u})$ is obtained after a little bit of algebra:

$$
\operatorname{ACF}_{I_{\text {Inv }}}(\mathbf{v})=\left\langle\mathrm{I}_{\text {Inv }}(\mathbf{u}) \cdot \mathrm{I}_{\mathrm{Inv}}(\mathbf{u}+\mathbf{v})\right\rangle
$$




$$
\begin{gathered}
\operatorname{ACF}_{I_{\text {Inv }}}(\mathbf{v})=\langle[-\mathrm{I}(\mathbf{u})+\operatorname{Max}[\mathrm{I}(\mathbf{u})]+\operatorname{Min}[\mathrm{I}(\mathbf{u})]] \cdot[-\mathrm{I}(\mathbf{u}+\mathbf{v})+\operatorname{Max}[\mathrm{I}(\mathbf{u}+\mathbf{v})]+\operatorname{Min}[\mathrm{I}(\mathbf{u}+\mathbf{v})]]\rangle(10 \\
\operatorname{ACF}_{I_{\text {Inv }}}(\mathbf{v})=\operatorname{ACF}_{\mathrm{I}}(\mathbf{v})+[\operatorname{Max}(\mathrm{I})+\operatorname{Min}(\mathrm{I})] \cdot[\operatorname{Max}(\mathrm{I})+\operatorname{Min}(\mathrm{I})-2\langle\mathrm{I}\rangle]
\end{gathered}
$$

Similarly, it can be shown that the NACFCs are related by:

$$
\mathrm{C}_{\mathrm{I}_{\mathrm{Inv}}}(\mathbf{v})=\mathrm{C}_{\mathrm{I}}(\mathbf{v})
$$

Consequently, any characteristic length obtained from $\mathrm{C}_{\mathrm{I}}(\mathbf{v})$ and associated to an object (the grains for instance) can also be obtained from $\mathrm{C}_{\mathrm{I}_{\mathrm{Inv}}}(\mathbf{v})$ and now associated to the background of $\mathrm{I}(\mathbf{u})$. Accordingly, $\mathrm{NACFC}_{\mathrm{I}}(\mathbf{v})$ cannot be used to determine object dimensions.

Single items or, more generally, loose aggregates of items constitute exceptions to this rule. Indeed, when the distance between the different items is large enough, there is a lag interval for which $C_{I}(\mathbf{v})$ is well approximated by the function corresponding to a single item.

To support this analysis we computed $\mathrm{C}_{\mathrm{I}}(\mathbf{v})$ for different $3 \mathrm{D}$ images $\left(750^{3}\right.$ pixels) representing spheres aggregates of different densities, i.e. different number of spheres per unit volume. The spheres diameters equal 20 pixels and Nsph, the number of spheres within the discretized volume, varies from 100 to 100000 (Fig. 12). To insure a random and homogeneous spatial distribution of the spheres, centres positions were computed using a random close packing generator based on [44]. This generator works as following:

1. Initialization: Nsph points are randomly placed within a cube. These points will be the centres of the Nsph spheres composing the packing, and the distance between the nearest centres will determine the spheres diameter. At this stage, due 
to the unconstrained random placement, the centres will be very heterogeneously distributed within the cube and the resulting spheres diameter will be very small.

2. Homogenisation phase: At each iteration, the two nearest spheres are symmetrically moved away along their centres line to a distance DI. During this phase, a large value is given to DI creating an efficient virtual shacking of the packing that will homogenise the distribution of the centres within the cube.

3. Densification phase: During this phase, DI is slowly decreased at each iteration, and the spheres diameter will gradually increase until convergence to a value corresponding to a dense random packing.

For more details on the parameters to be used during the different phases to effectively converge to a dense random packing, refer to [44].

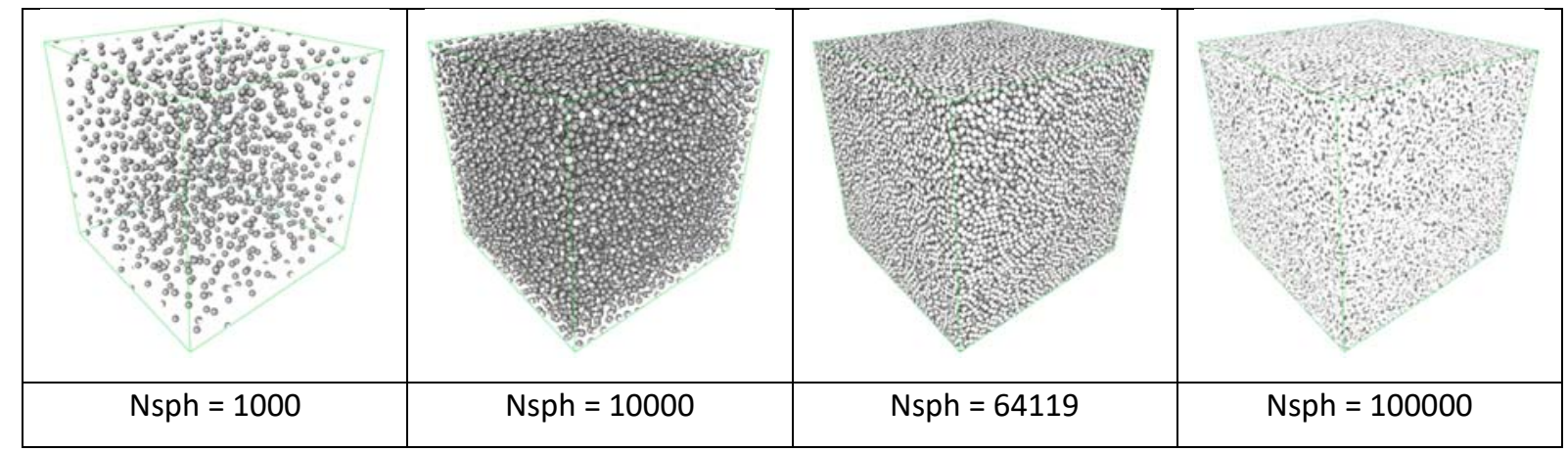

Figure 12: 3D rendering of some of the random spheres packings used in this work. For Nsph $<64119$ there is no overlap between the different spheres. For Nsph $=64119$, all spheres are in contact with their nearest neighbours. For Nsph $>64119$ there is some overlap between nearest spheres.

On Fig. 13.a are represented $\mathrm{C}_{\mathrm{I}}(\mathrm{v} . \mathbf{X})$, the NACFC in the direction of the axis $\mathbf{X}$, for 6 values of Nsph. It appears that up to $\mathrm{Nsph}=7500$ there is a distinct plateau for $\mathrm{v}$ moderately larger than $\delta$, the spheres diameter. The value of this plateau is given by the following formula: 


$$
-\frac{\langle\mathrm{I}(\mathbf{u})\rangle^{2}}{\left\langle\mathrm{I}(\mathbf{u})^{2}\right\rangle-\langle\mathrm{I}(\mathbf{u})\rangle^{2}}
$$

which corresponds to the value of the NACFC for $\mathrm{v}>\delta$ in the case of a single sphere. This means that each sphere is surrounded by an empty spherical domain having a radius larger than $2 \delta$. Table 6 gives, for the different values of Nsph, $\phi$, the volume fraction of the background in the corresponding three-dimensional discrete images, and MinDC, the minimum of the distances between sphere centres. For Nsph $>7500$, MinDC is larger than $2 \delta$, and interferences between neighbouring spheres begins. It can be noted that this occurs for very loose packing ( $\phi$ larger than $90 \%$ ).

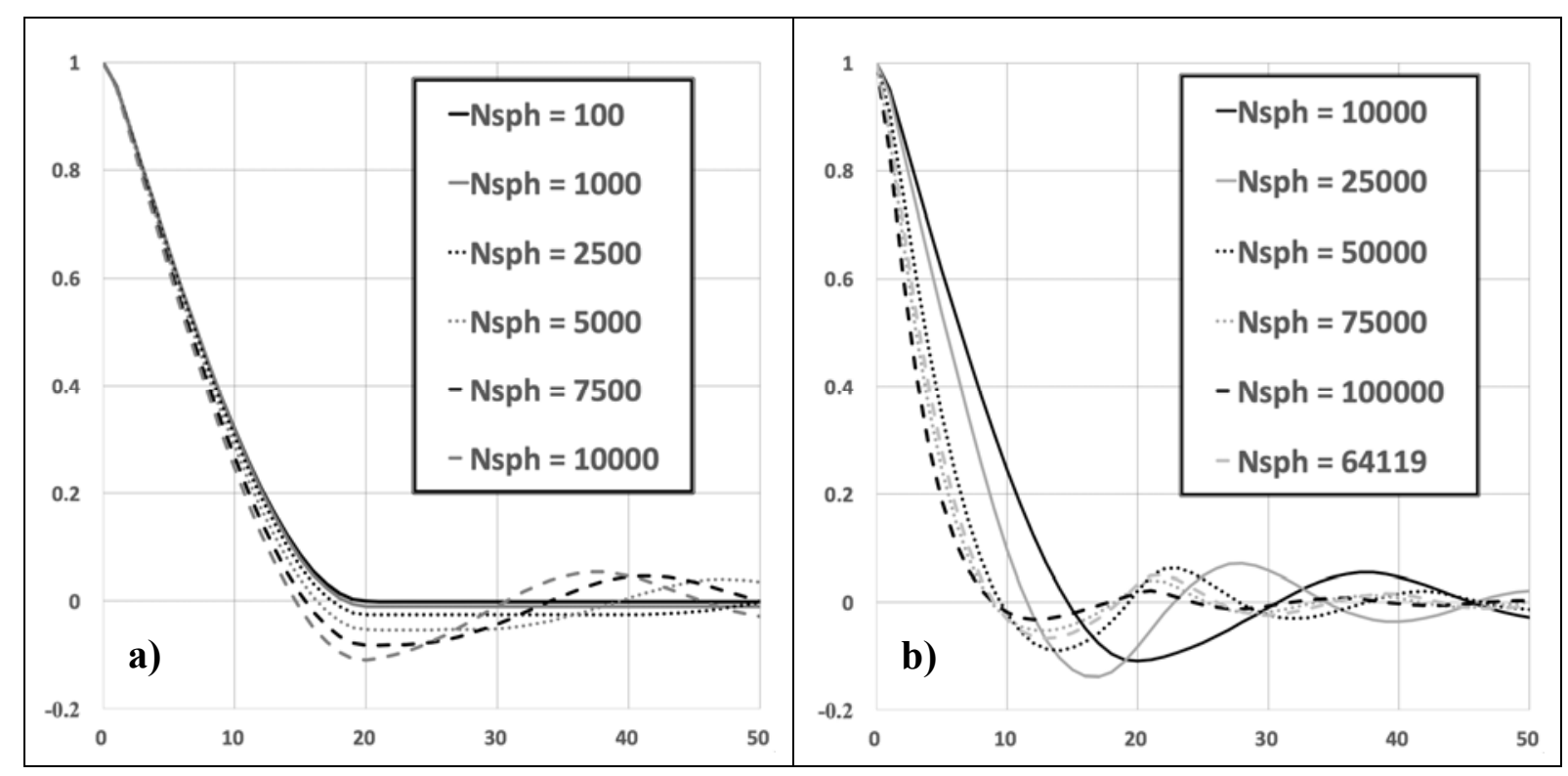

Figure 13: Evolution of the shape of NACFC with the number of spheres in the $3 D$

$$
\text { images. }
$$

When the number of spheres is increased, the shapes of the NACFC curves are modified (Fig. 13) with appearance of a minimum at a lag (La) where the anticorrelation of $\mathrm{I}(\mathbf{u})$ and $\mathrm{I}(\mathbf{u}+\mathbf{v})$ is maximum, and a maximum at a lag $(\mathrm{Lc})$ where the correlation of $\mathrm{I}(\mathbf{u})$ and $\mathrm{I}(\mathbf{u}+\mathbf{v})$ is maximum. Both lags are characteristics of the complete 
image, simultaneously taking into account spatial distributions of the objects (the spheres) and of the background.

Table 6: Evolution with Nsph, the number of spheres in the volume, of $\phi$, the porosity in $\%$, and MinDC, the minimum of the distances between sphere centres.

\begin{tabular}{|c|c|c|c|c|c|c|c|c|c|c|c|}
\hline Nsph & $\mathbf{1 0 0}$ & $\mathbf{1 0 0 0}$ & $\mathbf{2 5 0 0}$ & $\mathbf{5 0 0 0}$ & $\mathbf{7 5 0 0}$ & $\mathbf{1 0 0 0 0}$ & $\mathbf{2 5 0 0 0}$ & $\mathbf{5 0 0 0 0}$ & $\mathbf{6 4 1 1 9}$ & $\mathbf{7 5 0 0 0}$ & $\mathbf{1 0 0 0 0 0}$ \\
\hline $\boldsymbol{\phi}(\mathbf{\%})$ & 99.90 & 99.01 & 97.53 & 95.05 & 92.56 & 90.08 & 75.59 & 50.37 & 36.35 & 26.47 & 11.12 \\
\hline MinDC & 371.57 & 172.46 & 80.05 & 46.81 & 40.90 & 37.16 & 27.38 & 21.73 & 20.00 & 18.98 & 17.25 \\
\hline
\end{tabular}

Looking at Fig. 13 we observe that using any characteristic value of the NACFC (for instance 0.5 in [43]) to estimate the grains diameter is illusory. Indeed, the short-lag part of all the NACFC curves varies continuously with the sphere number. This is true for non-overlapping spheres aggregates $(\mathrm{Nsph}<=64119$, the value for having a random close packing) or for overlapping spheres aggregates.

$\mathrm{La}$ and Lc are characteristic distances related to the entire image and it is a priori not possible to distinguish the effects of its different components. This is unequivocally illustrated by the results of the series of tests summarized in Fig. 14. Seven discretized grey-level images $\left(750^{3}\right.$ pixels $)$ of aggregates comprising 25000 equal spheres were generated. In these aggregates the positions of the spheres centres were the same, but the spheres diameter varied between $\delta=20$ pixels and $\delta=32$ pixels by steps of 2 pixels.

On Fig. 13a are represented $C_{I}(v . X)$ for the 7 values of $\delta$. La and Lc increase with $\delta$, while both the anti-correlation and correlation levels decrease. La corresponds to the lag for which the average intersection between the spheres and the background is maximum. The positions of the sphere centres being constant (Fig. 14b), the variations of La can only be attributed to small variations of the distance between the spheres and the larger zones of the background. Lc represents the lag for which both background regions and the spheres reach maximum overlap. The increase of $\mathrm{Lc}$ is therefore also due to the variation of the distance between spheres and background regions, and the 
fact that it grows slightly faster than La can be explained by an increase of the distance between the nearest larger zones of the background.
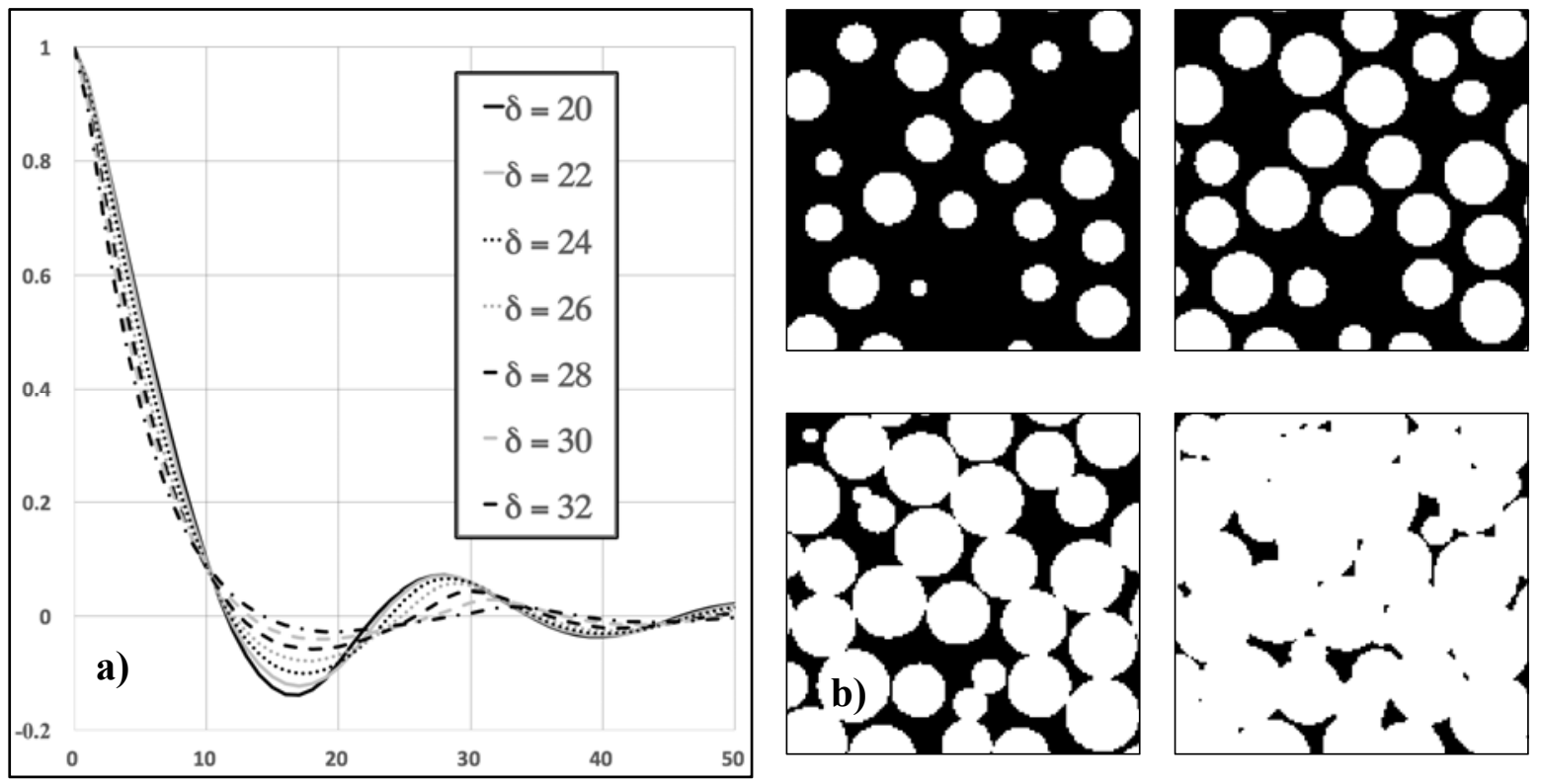

Figure 14: a) Evolution of the shape of NACFC with the diameter $\delta$ of the 25000

spheres composing the considered aggregates. b) Sections of the aggregates for $\delta=20$,

\section{4, 28, and 32 (left-right and up-down).}

\section{Appendix B: Tensor of inertia and ellipsoid}

The general equation of an ellipsoid centred at $(0,0,0)$ and aligned on the $\mathrm{X}, \mathrm{Y}, \mathrm{Z}$ axes is:

$$
\frac{x^{2}}{a^{2}}+\frac{y^{2}}{b^{2}}+\frac{z^{2}}{c^{2}}=1
$$

where $a, b$, and c are the principal semi-axes.

Assuming that the density of the ellipsoid is uniform, its moments of inertia with respect to the directions $\mathrm{X}, \mathrm{Y}$ and $\mathrm{Z}$ are given by the following expressions: 


$$
\begin{aligned}
& \mathrm{I}_{\mathrm{XX}}=\frac{\mathrm{m}}{5}\left(\mathrm{~b}^{2}+\mathrm{c}^{2}\right) \\
& \mathrm{I}_{\mathrm{YY}}=\frac{\mathrm{m}}{5}\left(\mathrm{a}^{2}+\mathrm{c}^{2}\right) \\
& \mathrm{I}_{\mathrm{ZZ}}=\frac{\mathrm{m}}{5}\left(\mathrm{a}^{2}+\mathrm{b}^{2}\right)
\end{aligned}
$$

Reciprocally, if $(\mathrm{X}, \mathrm{Y}, \mathrm{Z})$ is the coordinate system based on the principal directions of a moment of inertia tensor, the principal semi-axes of the ellipsoid having the same moment of inertia with respect of the directions $\mathrm{X}, \mathrm{Y}$ and $\mathrm{Z}$ are given by the following expressions:

$$
\begin{aligned}
& a=\sqrt{\frac{5}{2 m}\left(-I_{X X}+I_{Y Y}+I_{Z Z}\right)} \\
& \mathrm{b}=\sqrt{\frac{5}{2 \mathrm{~m}}\left(\mathrm{I}_{\mathrm{XX}}-\mathrm{I}_{\mathrm{YY}}+\mathrm{I}_{\mathrm{ZZ}}\right)} \\
& c=\sqrt{\frac{5}{2 m}\left(I_{X X}+I_{Y Y}-I_{Z Z}\right)}
\end{aligned}
$$

When presenting the autocorrelation function, two definitions were introduced for the degree of anisotropy; DA_Inertia and DA_Ellips. Considering an ellipsoid described by Eq. 12 for which $\mathrm{a} \geq \mathrm{b} \geq \mathrm{c}$ and using the relations 13 and 14, the following expressions are obtained:

$$
\begin{gathered}
\text { DA_Inertia }=\frac{\mathrm{I}_{\mathrm{Zz}}}{\mathrm{I}_{\mathrm{XX}}} \\
\text { DA_Ellips }=\frac{\mathrm{a}}{\mathrm{c}} \\
\text { DA_Inertia }=\frac{(\text { DA_Ellips })^{2}+\alpha}{\alpha+1} \text { with } \alpha=\left(\frac{\mathrm{b}}{\mathrm{c}}\right)^{2}
\end{gathered}
$$

$\alpha$ is larger than 1, and depending on its value, DA_Inertia is larger or smaller than DA_Ellips.

If we take the case of needle (e.g. $\mathrm{a}=10$, and $\mathrm{b}=\mathrm{c}=1)$, we obtain DA_Ellips $=$ 10 and DA_Inertia $=50.5$, and in the case of a disc (e.g. $\mathrm{a}=\mathrm{b}=10$, and $\mathrm{c}=1)$, we get DA_Ellips $=10$ and Izz/Ixx $\sim 1.98$. This implies that the objects in Fig. 1 have the shape 
of discs rather than that of needles, as expected from their definition. Trivially, in the case of a sphere (i.e., $\mathrm{a}=\mathrm{b}=\mathrm{c})$, DA_Ellips $=$ DA_Inertia $=1$.

\section{Appendix c: Permeability eigenvalues and eigenvectors}

\begin{tabular}{|c|c|c|c|c|c|}
\hline \multicolumn{6}{|c|}{ Sub-sample I $\mathrm{I}_{\mathrm{EL}}$} \\
\hline \multirow{2}{*}{\multicolumn{2}{|c|}{ Eigenvalues }} & \multicolumn{4}{|c|}{ Eigenvectors } \\
\hline & & & $A$ & B & C \\
\hline$\tau_{1}$ & 3.208 & $v_{1}$ & 0.99998 & $-2.756 \mathrm{E}-3$ & $-4.501 \mathrm{E}-3$ \\
\hline$\tau_{2}$ & 1.714 & $v_{2}$ & $4.223 \mathrm{E}-3$ & 0.92935 & 0.36918 \\
\hline$\tau_{3}$ & 0.336 & $v_{3}$ & $3.166 \mathrm{E}-3$ & -0.36919 & 0.92935 \\
\hline \multicolumn{6}{|c|}{ Sub-sample Cord1 } \\
\hline \multirow{2}{*}{\multicolumn{2}{|c|}{ Eigenvalues }} & \multicolumn{4}{|c|}{ Eigenvectors } \\
\hline & & & $\mathrm{A}$ & $\mathrm{B}$ & $\mathrm{C}$ \\
\hline$\tau_{1}$ & 7.898 & $v_{1}$ & $-5.596 \mathrm{E}-2$ & 0.99571 & $-7.366 \mathrm{E}-2$ \\
\hline$\tau_{2}$ & 7.105 & $v_{2}$ & 0.96822 & $3.610 \mathrm{E}-2$ & -0.24748 \\
\hline$\tau_{3}$ & 6.944 & $v_{3}$ & 0.24376 & $8.517 \mathrm{E}-2$ & 0.96609 \\
\hline \multicolumn{6}{|c|}{ Sub-sample Cord2 } \\
\hline \multirow{2}{*}{\multicolumn{2}{|c|}{ Eigenvalues }} & \multicolumn{4}{|c|}{ Eigenvectors } \\
\hline & & & A & $B$ & C \\
\hline$\tau_{1}$ & 3.916 & $v_{1}$ & $7.765 \mathrm{E}-2$ & 0.99161 & -0.10331 \\
\hline$\tau_{2}$ & 3.098 & $v_{2}$ & $-6.421 \mathrm{E}-2$ & 0.10838 & 0.99203 \\
\hline$\tau_{3}$ & 2.881 & $v_{3}$ & 0.99491 & $-7.040 \mathrm{E}-2$ & $7.208 \mathrm{E}-2$ \\
\hline \multicolumn{6}{|c|}{ Sub-sample Cord3.1 } \\
\hline \multirow{2}{*}{\multicolumn{2}{|c|}{ Eigenvalues }} & \multicolumn{4}{|c|}{ Eigenvectors } \\
\hline & & & $A$ & $B$ & $\mathrm{C}$ \\
\hline$\tau_{1}$ & 0.845 & $v_{1}$ & $4.800 \mathrm{E}-2$ & 0.99400 & $-9.824 \mathrm{E}-2$ \\
\hline$\tau_{2}$ & 0.772 & $v_{2}$ & 0.88500 & $3.282 \mathrm{E}-3$ & 0.46558 \\
\hline$\tau_{3}$ & 0.597 & $v_{3}$ & -0.46312 & 0.10929 & 0.87953 \\
\hline \multicolumn{6}{|c|}{ Sub-sample Cord3.2 } \\
\hline \multirow{2}{*}{\multicolumn{2}{|c|}{ Eigenvalues }} & \multicolumn{4}{|c|}{ Eigenvectors } \\
\hline & & & $A$ & B & C \\
\hline$\tau_{1}$ & 1.044 & $v_{1}$ & $-7.458 \mathrm{E}-3$ & 0.98976 & 0.14253 \\
\hline$\tau_{2}$ & 0.885 & $v_{2}$ & 0.83933 & $-7.128 \mathrm{E}-2$ & 0.53893 \\
\hline$\tau_{3}$ & 0.723 & $v_{3}$ & -0.54358 & -0.12364 & 0.83020 \\
\hline \multicolumn{6}{|c|}{ Sub-sample Cord4.1 } \\
\hline \multirow{2}{*}{\multicolumn{2}{|c|}{ Eigenvalues }} & \multicolumn{4}{|c|}{ Eigenvectors } \\
\hline & & & $\mathrm{A}$ & B & $\mathrm{C}$ \\
\hline$\tau_{1}$ & 2.116 & $v_{1}$ & $7.030 \mathrm{E}-2$ & 0.99746 & $-1.118 \mathrm{E}-2$ \\
\hline$\tau_{2}$ & 1.909 & $v_{2}$ & 0.98855 & $-7.116 \mathrm{E}-2$ & -0.19258 \\
\hline$\tau_{3}$ & 1.797 & $v_{3}$ & 0.13350 & $1.676 \mathrm{E}-3$ & 0.98122 \\
\hline \multicolumn{6}{|c|}{ Sub-sample Cord4.2 } \\
\hline & glues & & & ectors & \\
\hline
\end{tabular}




\begin{tabular}{|c|c|c|c|c|c|}
\hline & & & $A$ & $B$ & C \\
\hline$\tau_{1}$ & 2.133 & $v_{1}$ & $-8.971 \mathrm{E}-2$ & 0.99591 & $-9.824 \mathrm{E}-2$ \\
\hline$\tau_{2}$ & 1.927 & $v_{2}$ & 0.97713 & $9.018 \mathrm{E}-2$ & 0.46558 \\
\hline$\tau_{3}$ & 1.802 & $v_{3}$ & 0.19280 & $6.353 \mathrm{E}-3$ & 0.87953 \\
\hline \multicolumn{6}{|c|}{ Sub-sample SiC } \\
\hline \multirow{2}{*}{\multicolumn{2}{|c|}{ Eigenvalues }} & \multicolumn{4}{|c|}{ Eigenvectors } \\
\hline & & & $A$ & $\mathrm{~B}$ & $\mathrm{C}$ \\
\hline$\tau_{1}$ & 0.699 & $v_{1}$ & 0.97144 & 0.22907 & $-6.196 \mathrm{E}-2$ \\
\hline$\tau_{2}$ & 0.651 & $v_{2}$ & -0.23597 & 0.96012 & -0.14994 \\
\hline$\tau_{3}$ & 0.625 & $v_{3}$ & $2.514 \mathrm{E}-2$ & 0.16027 & 0.98675 \\
\hline
\end{tabular}

